DOI: https://doi.org/10.24297/jam.v18i.8746

\author{
If the Propagator of QED Were Reversed, The Mathematics of Nature Would be Much Simpler \\ Jeffrey H. Boyd, \\ Retired \\ 57 Woods Road, Bethany, CT, 06524-3103 USA \\ Jeffreyhboyd@gmail.com \\ May 19, 2020
}

\begin{abstract}
:
In Quantum ElectroDynamics (QED) the propagator is a function that describes the probability amplitude of a particle going from point A to B in a certain amount of time. It integrates across the infinite paths of Feynman's path integral approach. We propose a reverse propagator (R-propagator) that, prior to the particle's emission, integrates across every possible path from B to A. Wave function collapse occurs at A when the particle randomly chooses only one of incident paths to follow backwards with a probability of one, so it inevitably strikes detector B. The propagator and R-propagator both calculate the same probability amplitude, using exactly the same equations. The R-propagator solves a contradiction inside QED, namely QED says a particle must take EVERY path from A to B. With our model the particle only takes one path. The R-propagator had already integrated across every path. We propose that this tiny, infinitesimal change of direction from propagator to R-propagator would vastly simplify the mathematics of Nature. Many experiments that currently describe the quantum world as weird, change their meaning and no longer say that. The quantum world looks and acts like the classical world of everyday experience.
\end{abstract}

\title{
Subject Classification
}

Mathematics Subject Classification 81Q30: Feynman integrals and graphs. Library of Congress Classification sh85109459: quantum electrodynamics

\section{Introduction}

This article proposes the existence of a reverse propagator (R-propagator) that is exactly like Richard Feynman's propagator except that it integrates in the opposite direction (from B to A), prior to the emission of the particle. The particle randomly chooses among the incident paths, then follows one of them back to B with a probability of one. The equations for both R-propagator and propagator are identical. A change of direction solves a long list of otherwise insoluble problems. It is a novel way of thinking.

In QED the propagator (or kernel) is a function that calculates the probability amplitude of a particle going from point A to B in a certain amount of time. QED integrates across every possible path. It is the most accurate science that humans ever possessed. It does, however, have a well-known flaw, namely the requirement that every particle must take EVERY path from point A to B. Common sense tells us that each individual particle only takes one route.

The proposed R-propagator and particle interact in the way shown in Figure 1. We define an R-propagator that, prior to when the particle is emitted, includes EVERY path from point $\mathrm{B}=(\mathrm{t} 1, \mathrm{q} 1)$ to $\mathrm{A}=(\mathrm{t} 2, \mathrm{q} 2)$. Like paths you walk on in the woods, the paths of QED are bi-directional, with time always going forwards (i.e. $t 3>t 2>t 1$ ), never backwards. The R-propagator travels from B to A before the gun is fired. The particle, as it surveys the infinity of incident trajectories, randomly chooses only one trajectory to follow backwards. Wave function collapse occurs at point $A=(t 2, q 2)$ as the particle leaves the gun. Between A and B the particle follows its chosen path with a probability of one. Neither in QED nor in our model do the paths impart any energy to a particle. Paths don't push or pull a particle; they do no work.

This approach preserves the accuracy of QED (because the R-propagator integrates across every path), but eliminates the logical flaw (one particle traverses only one path). This article gives you the mathematics of a R-propagator, and demonstrates that it dispels many forms of quantum weirdness. For example, with R-propagators the Schrődinger's cat paradox is resolved, because wave function collapse occurs when a particle is emitted from point A, not when it is detected at point $\mathrm{B}$.

Our model takes roughly twice as long as Feynman's model, and includes twice the distance. Our attention moves from $\mathrm{B}$ to $\mathrm{A}$, then back to $\mathrm{B}$. On the first leg of that journey every possible path is included in our equations, which are identical to the equations of QED. The total probability amplitude of the particle going from A to B is the same with our theory as with Feynman's.

Our model answers a question of John von Neumann, "How does the randomness get into QM when the equations all focus on predictability?" Answer: "It come from the particle, which, as it is about to leave the gun, randomly selects which of the incident paths to follow back to detector B with a probability of one.." 


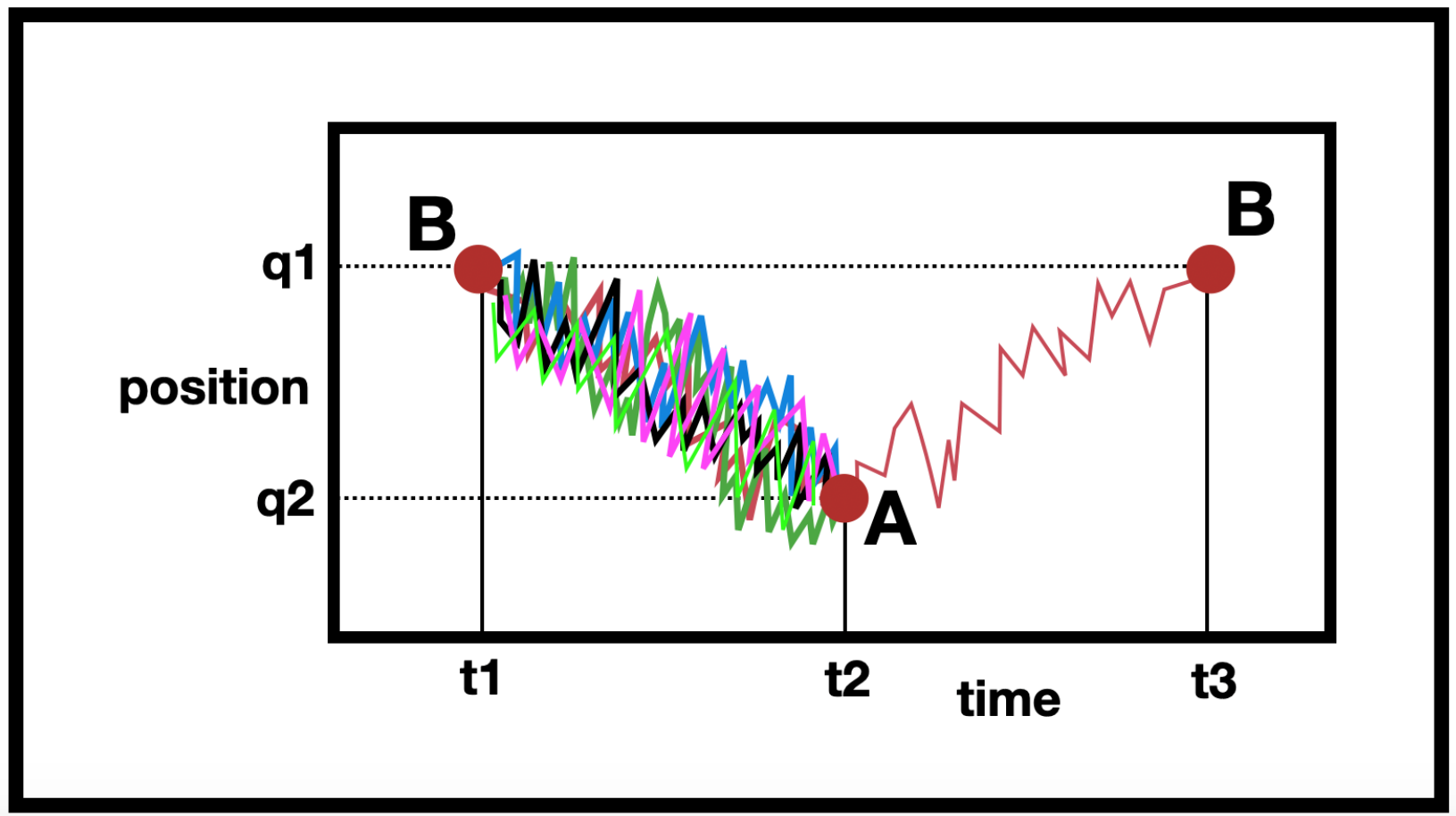

Figure 1: The R-propagator model describes the probability amplitude of a particle going from point A to point B. Time always moves forwards in this diagram: $\mathrm{t} 3>\mathrm{t} 2>\mathrm{t} 1$. If the reader has trouble understanding the layout of this diagram, we will explain it in Fig. 2. Our model calculates the same total amplitude as Feynman's, but gets there in a way the reader never imagined. First, the R-propagator integrates across all possible routes from the detector at point $\mathrm{B}=(\mathrm{t} 1$, q1) to $\mathrm{A}=(\mathrm{t} 2, \mathrm{q} 2)$ before the gun is fired (before the particle is emitted). Wave function collapse occurs at $\mathrm{A}$ when the particle makes a random choice among incident trajectories. The particle then follows that one path back to $B=(t 3, q 1)$, with an amplitude of one.

\subsection{Two solutions to the tangled QED equations}

The purpose of the path integral approach is to accurately calculate the amplitude of a particle traveling from point A to $\mathrm{B}$ in a certain amount of time. But let's turn the theory around. Instead of defining the probability amplitude as the output, suppose we defined it as the input, and we define the propagator as the output.

This is analogous to the following puzzle. Everyone else is trying to solve for $\mathrm{y}$ in the equation $\sin ^{2} x=y$ for $-\pi / 2 \leq x \leq \pi / 2$, but you are interested in the inverse problem: trying to solve for $x$ in $\sin ^{2} x=y$. The conventional wisdom says that the answer to the first equation is $0 \leq y \leq 1$ and therefore the answer to your puzzle is $0 \leq x \leq \pi / 2$. However you say, "There is another solution that works equally well, $-\pi / 2 \leq x \leq 0$." People tell you no one cares, because they already have a solution that works.

You ask the question, "If there are two solutions, and Nature only uses one, then how do you know whether your ' $x$ ' is the one that Nature uses? From a practical point of view there is a mountain of evidence that my ' $x$ ' is closer to Nature than yours."

This article proposes that both the propagator and R-propagator use exactly the same equations, but from a practical viewpoint the R-propagator is better. The propagator produces quantum weirdness. The R-propagator doesn't.

\subsection{Guide to our diagrams}

If the reader asks, "How does this article discover a second solution, previously unknown, to the equations of QED?" The answer is, "Because we re-arranged the graph paper, as you can see in Fig. 2."

Fig. 2 explains the diagrams used in this article. The first square on the left (labelled with a blue "1") has the format of a simple Feynman path integral diagram with a curve traveling from $A=(t 1, q 1)$ to $B=(t 2$, q2), where " $t$ " is time and " $q$ " is position. The second square has changed the labels: "A" became "B" and "B" became "A." Nothing else changed. Square 2 calls our attention to the fact that the equations of the path integral approach are time reverse symmetric. 
Throughout his life Feynman loved the idea of time reverse symmetry. He believed that time routinely went both directions. He taught that an electron and positron were the same particle, one with time going forwards, the other backwards in time. His first publication was about time reversal (Wheeler \& Feynman 1945). Nevertheless, in this article time will always flow forwards: $\mathrm{t} 3>\mathrm{t} 2>\mathrm{t} 1$, in order to avoid confusion.

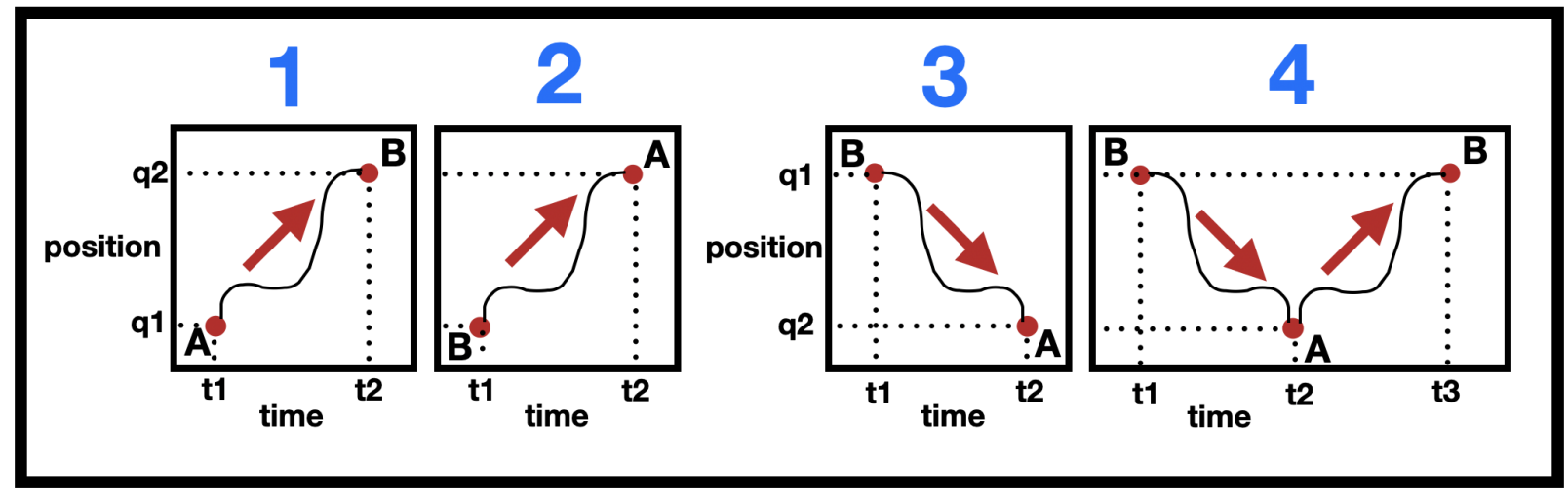

Figure 2: These four rectangles explain the diagrams used in this article.

In the third square we moved the vertical locations of points B and A. Point B was elevated vertically to position (t1, q1) and point A was dropped straight down to position (t2, q2). Nothing else changed. Our plan is to integrate in the direction of the red arrow, from point $B=(t 1, q 1)$ to $A=(t 2, q 2)$.

The fourth rectangle (Fig. 2 right) is twice as wide, and summarizes the model we will use. There are two stages. On the left, with no particle involved, the R-propagator starts at $\mathrm{B}=(\mathrm{t} 1, \mathrm{q} 1)$ and moves to $\mathrm{A}=(\mathrm{t} 2$, q2). Time always goes forwards. When the R-propagator reaches the gun at point $\mathrm{A}=(\mathrm{t} 2, \mathrm{q} 2)$, the particle randomly selects one of the incident pathways to follow backwards, with a probability of one, to point $B=(t 3, q 1)$.

The pathway from $\mathrm{A}$ in the center of rectangle 4, up and to the right to the detector at point $\mathrm{B}$, has an amplitude of one, as we will explain below. Nevertheless, the total amplitude of the particle traveling from A to B is the same with our scheme as with Feynman's path integral scheme. Lewis E. Little taught us the idea that a particle follows a zero energy pathway backwards with a probability of one.

\subsection{The central theme of this article}

Here is a thumbnail sketch of what this article says. The equations of our system are, with one exception (see next paragraph), the same as the equations of QED. The R-propagator and the propagator calculate exactly the same amplitude for a particle to travel from A to B in a certain amount of time. QED could run equally well with either engine. Which engine does Nature use? There is abundant evidence that Nature uses R-propagators, not propagators. That evidence is in the form of eliminating quantum weirdness.

The exception, where our equations differ from those of QED, is that our approach to integration is backwards. QED integrates from A to B. We integrate from B to A. Given Feynman's love of time reverse symmetry, he would not regard this as a problem. What Feynman regards as "time reversal" we regard as time going forwards, but Nature using waves that travel in the opposite direction as particles.

We focus on free particles because there we can see whether particles travel in one direction, following R-propagators that travel in the opposite direction. This focus on "Which direction?" would not be visible if we were studying harmonic oscillators, or particles bound inside an atom. Traditionally terms like " $\mathrm{V}$ " for potential energy are omitted from equations for a free particle. We however will include potential energy terms so that our equations are more generizable.

\section{R-propagator defined}

We define an R-propagator to be a function that describes the probability amplitude of a particle going from point $\mathrm{A}$ to B in a certain amount of time. It differs from Feynman's propagator because the R-propagator summarizes (integrates across) all paths moving centripetally toward the gun (the left hand side of Figs. 1 and 4) before the particle leaves the gun. From a mathematical viewpoint this is, with one exception, an insignificant difference. The exception is shown in Fig. 3. 


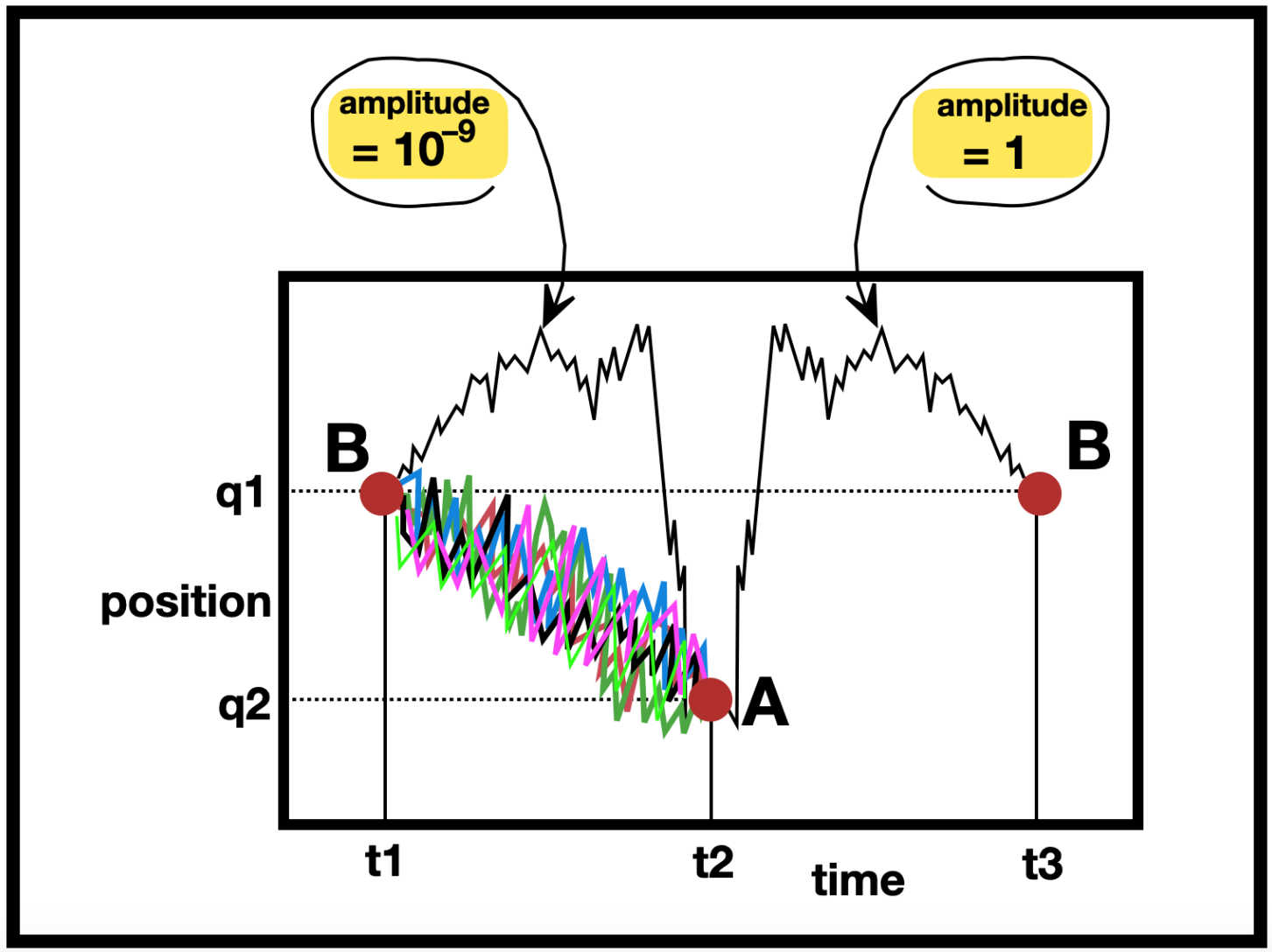

Figure 3: The R-propagator model has this unusual feature. An obscure path on the left (black line) has an infinitesimal amplitude $\left(10^{-9}\right)$. However, the particle might select that trajectory one in a billion billion times $\left(10^{-9}\right)^{2}$, in which case the particle follows that trajectory to the right with a probability of one. Time always moves forwards in this diagram: $\mathrm{t} 3>\mathrm{t} 2>\mathrm{t} 1$. This peculiar feature does not change the total probability amplitude of the particle going from A to B in the R-propagator model, because the R-propagator function has already integrated across ALL pathways. The equations below focus on the left side of this diagram (from B to A), whereas the randomness of QM comes from the right side of this diagram (from $\mathrm{A}$ to $\mathrm{B}$ ).

The left half of Fig. 3 shows all trajectories from point $B=(t 1, q 1)$ to $A=(t 2, q 2)$, which is based on exactly the same math as that of the propagators (see below). At the gun [point $A=(t 2, q 2)$ ] the particle makes a random choice of which incident pathway to respond to. It is like deciding to marry one partner among many suitors, then remaining faithful to that spouse thereafter. That choice causes wave function collapse. All the pathways that are not selected become irrelevant. The particle then follows its chosen pathway backwards to the detector at point $B=(t 3, q 1)$ with an amplitude of one. That does not change the total probability amplitude based on all pathways. Despite the weird feature shown in Fig. 3, R-propagators and propagators yield identical mathematics. For example, the amplitude for a particle going from point $\mathrm{A}=(\mathrm{t} 2, \mathrm{q} 2)$ to $\mathrm{B}=(\mathrm{t} 3, \mathrm{q} 1)$ following the black pathway would be the same: $10^{-9}$ in both cases.

Like Feynman's propagator, the central focus of our equations is on the phase, and specifically on the variable $\mathrm{S}$ (action) in the exponent that computes the phase. The black trajectory in Fig. 3 would have a phase that is very divergent from the phase of the R-propagator, and that would make that trajectory unlikely to be chosen (large action S).

We borrow much of the mathematical argument below from Konka Ravi Teja, but we present a mirror image of Ravi Teja's thinking because he is interested in paths from A to B, whereas we are interested in paths from B to A. (Ravi Teja 2017). He never thought about R-propagators traveling in what he would regard as the "wrong direction."

Prior to this article no one ever spoke of, or even thought about R-propagators. Not even Lewis E. Little. It is an idea that popped into this author's imagination in April 2020 when he was watching a YouTube video by Andrew Dotson (2019). The author had been moving toward this idea over the past five years (Boyd 2015b, 2015g).

The ball game is over once the particle leaves the gun. Wave function collapse occurs at the gun, not at the detector. That is what it means to say, on the right side of Fig. 3, that the "amplitude $=1$." This difference in the timing of wave function collapse is a central difference between this model and garden variety QM. 
The mathematics of a R-propagator, defined below, is the same for a propagator. Even if some feature of a propagator amplitude in someone else's mathematical system corresponds to the negative (i.e. a minus sign) of the amplitude of an R-propagator, that would not matter because to obtain a probability you square the amplitude, thereby obliterating the difference between positive and negative signs. However, this author doesn't see a need for a minus sign because time is always going forwards ( $\mathrm{t} 3>\mathrm{t} 2>\mathrm{t} 1)$, never backwards, in our scheme.

As noted in section 1.2, a central goal of this article is to convince the reader that R-propagators and propagators have the same equations, and therefore QED can equally well run with either engine.

\subsection{Deriving the math for R-propagators in QED}

Feynman's propagators arose from his high school days when his physics teacher, Abram Bader, taught him the calculus of variations. It was love at first sight. According to Joseph Lagrange and Leonhard Euler, the action S was a time integral:

$$
S=\int_{t 1}^{t 2}(\text { Kinetic Energy - Potential Energy }) d t
$$

Nature is lazy, and usually follows the path with the least action. In a single dimensional, single particle situation

$$
S=\int_{t 1}^{t 2}\left[\frac{m}{2}\left(\frac{d x(t)}{d t}\right)^{2}-V(x(t))\right] d t .
$$

\section{$\bigwedge$ Lagrangian}

The propagator is Green's function for the time dependent Schrődinger equation (Dotson 2019). How does a particle know which is the path of least action? Feynman requires that the particle must take EVERY possible path.

The mathematics used to treat quantum phenomena are analogous to the mathematics used to treat the statistical physics of temperature. To exploit that similarity Ravi Teja treats the variable $\hbar$ as analogous to temperature. This allows him to invoke a robust body of mathematics, so as to solve problems in quantum field theory. He uses the statistical temperature of a cloud of particles in Euclidean time, as a metaphor for the quantum electrodynamics problem in real time, where the term "metaphor" refers to a mathematical trick. Sometimes he speaks of a "cluster of particles" other times he speaks of a "cluster of trajectories," because in this model they are analogous.

Table 1

A quantum system in real time is analogous to (similar math) a Thermostat in Euclidean time

\begin{tabular}{|l|l|l|l|}
\hline \multicolumn{2}{|c|}{ QM at finite temperature } & is analogous to & A statistical ensemble of particles or paths \\
\cline { 1 - 2 } & Temperature $\quad \mathrm{T}_{\text {quantum }}$ & is analogous to & \\
\hline 2. & Planck's constant $\hbar$ & is analogous to & The period $\tau_{\beta}$ \\
\hline 3. Quantum fluctuations of a single particle & is analogous to & Thermal fluctions of ensemble of particles \\
\hline
\end{tabular}

In Table 1 Ravi Teja compares a quantum particle (on the left) to the statistical ensemble of a thermostat (on the right). He postulates that they are similar to one another. The first row shows that finite temperature in real time is equivalent to period in Euclidean time. The second row shows that $\hbar$ is analogous to statistical temperature. There is an analogy between QM at finite temperature in real time and a classical statistical ensemble of trajectories in Euclidean time.

\subsubsection{A quantum mathematical approach}

The Hamiltonian of a one dimensional, non-relativistic particle is

$$
H=\frac{p^{2}}{2 m}+V(q)
$$

where $\mathrm{p}$ is momentum, $\mathrm{m}$ is the mass, $\mathrm{q}$ the position. $\mathrm{V}$ is the potential energy, which is stratified into energy eigenstates. The position eigenstates $|q\rangle$ are such that $Q|q\rangle=q|q\rangle$.

Evolution of the quantum states is given by the evolution operator

$$
U(t)=\exp \left(\left(\frac{t}{i \hbar}\right) H\right)
$$

If at $t=0$ the particle is in state $\left|\Psi_{0}\right\rangle$ then at a later time $|\Psi(t)\rangle=U(t)\left|\Psi_{0}\right\rangle$ 


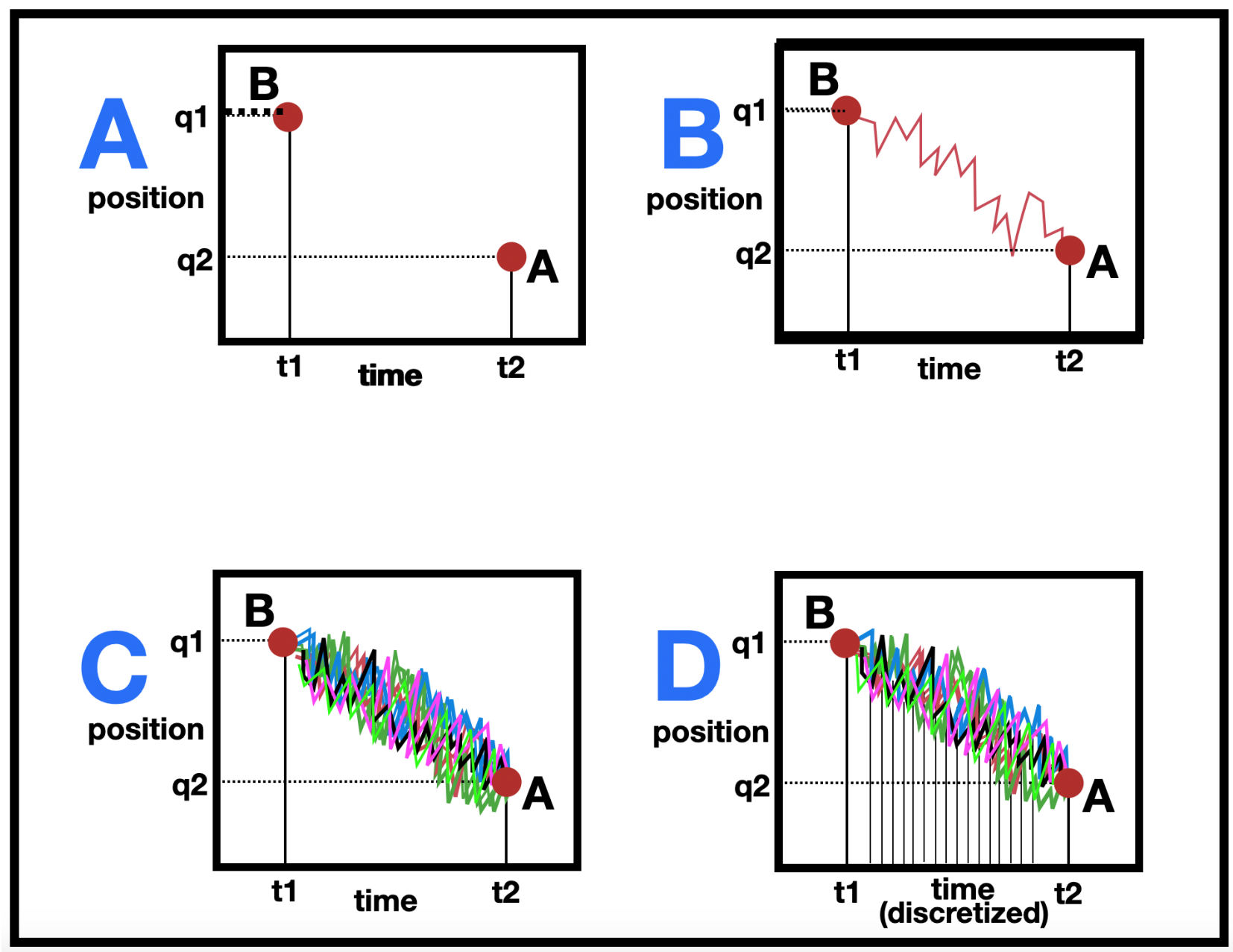

Figure 4: Four graphs showing a variety of paths used in the R-propagator model: A. boundary conditions (at $\mathrm{t} 1$ the particle is at $\mathrm{q} 1$ which is point $\mathrm{B}, \&$ at $\mathrm{t} 2$ it is at $\mathrm{q} 2$ which is point $\mathrm{A}$ ); B. One possible path from q1 to q2; C. All possible histories in real time; D. Time is discretized and then we take the limit as the width of each slice $\Delta t \longrightarrow 0$.

\subsubsection{Feynman's approach}

We want to integrate across all pathways from B to A. How many are there? The number of paths from B to A is identical to the number of paths from A to B. Feynman declares that "the number of paths is a high order of infinity" (Feynman \& Hibbs 1965). Infinities are a notorious problem in quantum field theories. In this case we can deal with the infinity of paths by knowing how to normalize our equations. Feynman says the normalizing factor is $\mathcal{A}^{-N}$ where

$$
\mathcal{A}=\left(\frac{2 \pi i \hbar \Delta t}{m}\right)^{1 / 2}
$$

We define the R-kernel "K" (i.e. R-propagator) to be:

$$
K(a, b)=\lim (\Delta t \rightarrow 0) \frac{1}{\mathcal{A}} \int_{t_{1}}^{t_{2}} \ldots \iint_{t_{1}}^{t_{2}} e^{(i / \hbar) S(q)} \frac{d x_{2}}{\mathcal{A}} \frac{d x_{1}}{\mathcal{A}} \ldots \frac{d x_{N-1}}{\mathcal{A}}
$$

where $\Delta t$ is the width of each slice of time in Fig. 4D. We will use a variable named "script $\mathcal{D}$ " to symbolize Eq. 5's integration across all the pathways. This variable is used in Eq. 7. The R-path integral is defined:

$$
\begin{gathered}
K(q 2, t 2, q 1, t 1)=\left\langle q_{2}\right|\left(U\left(t_{2}-t_{1}\right)\left|q_{1}\right\rangle\right. \\
=\lim (\Delta t \rightarrow 0)\left\{\int_{t_{1}}^{t_{2}} \mathcal{D}[q(s)] \exp \left(\frac{i}{\hbar} S(q)\right)\right\}
\end{gathered}
$$




\section{$\bigwedge \operatorname{script} \mathcal{D}$ (refers to Eq. 5)}

where $\mathbf{S}(\mathbf{q})$ is the action of the histories:

$$
S(q)=\int_{t_{1}}^{t_{2}} d s\left[\frac{m}{2}\left(\frac{d q}{d s}\right)^{2}-V\left(q_{s}\right)\right]
$$

\section{$\bigwedge$ Lagrangian see Eq.1}

\subsubsection{The statistics of thermal systems}

We now draw on a different body of mathematical physics, because our system is sufficiently similar that we can borrow some of the mathematical techniques of statistical mechanics. A "canonical ensemble" is the statistical ensemble that represents the possible states of a mechanical system in thermal equilibrium with a heat bath at a fixed temperature. The system can exchange energy with the heat bath, so the states of the system will differ in total energy. Later we will draw an analogy between an ensemble of particles and an ensemble of different trajectories.

Ravi Teja imagines our simple quantum particle to be at equilibrium with a large thermostat (i.e. a "canonical ensemble"), which has a lot of particles in various states of energy. Since our particle is coupled with this thermostat, we can only consider the state of our particle in a statistical sense of estimating the mixed thermal state of that canonical ensemble, which can be described by a density matrix $\rho$.

It has a simple form called the Gibbs state:

$$
\rho=\frac{1}{Z} \exp (-\beta H)
$$

where $\mathrm{Z}$ is a normalization variable, $\mathrm{H}$ is the Hamiltonian, and

$$
\beta=\frac{1}{k_{B} T}
$$

and $k_{B}$ is the Boltzmann constant, and $\mathrm{T}$ is the temperature. The normalization factor $\mathrm{Z}$ is called the "partition function" which is defined:

$$
Z=\operatorname{Tr}(\exp (-\beta H)) .
$$

which is defined such that $\operatorname{Tr}(\rho)=1$. If $\mathrm{A}$ is any observable, then at temperature $\mathrm{T}$,

$$
\langle A\rangle_{T}=\operatorname{Tr}(\rho A)
$$

\subsubsection{Euclidean time (i.e. imaginary time)}

Felix Bloch showed that we can view a quantum system at thermal equilibrium as analogous to a quantum system in Euclidean time (Bloch, 1957). Euclidean time means imaginary time. This allows us to make a Wick rotation of Minkowski coordinates $(-,+,+,+)$ into Euclidean coordinates $(+,+,+,+)$ that are easier to work with. It takes oscillatory functions and converts them into dampening functions (Gaussians) that are more manageable.

To switch to Euclidean time, we replace time $(\mathrm{t})$ with imaginary time $(-i \tau)$ :

$$
\begin{array}{r}
\exp (-\beta H)=U(i \tau) \\
\text { such that } \frac{\tau}{\hbar}=\beta=\frac{1}{k_{B} T}
\end{array}
$$

We can redefine the evolution operator $\mathrm{U}$ as follows:

$$
U(t)=\sum_{n} e^{(t / i \hbar) E_{n}}|n\rangle\langle n|
$$

We can see that $e^{(t / i \hbar) E_{n}}$ is convergent only if imaginary time is less than zero. So that is our only constraint: that imaginary time must be less than zero.

To find the path integral representation for this object: $U(-i \tau)=U_{E}(\tau)$ where "E" means "Euclidean", we construct the path integral at each of the steps, and replace $\Delta t$ with $-i \Delta \tau$. We need to know the matrix element $K(q 2, t 2, q 1, t 1)=\left\langle q_{2}\right|\left(U\left(t_{2}-t_{1}\right)\left|q_{1}\right\rangle\right.$ at two very short times $\mathrm{t} 1$ and $\mathrm{t} 2$ and for two very short distances. So we replace each of the tiny time steps by imaginary $\tau$ steps, and run through the machinery. This results in:

$$
K_{E}\left(q_{2}, \tau_{2}, q_{1}, \tau_{1}\right)=\left\langle q_{2}\left|\left(U_{E}\left(\tau_{2}-\tau_{1}\right)\right)\right| q_{1}\right\rangle
$$




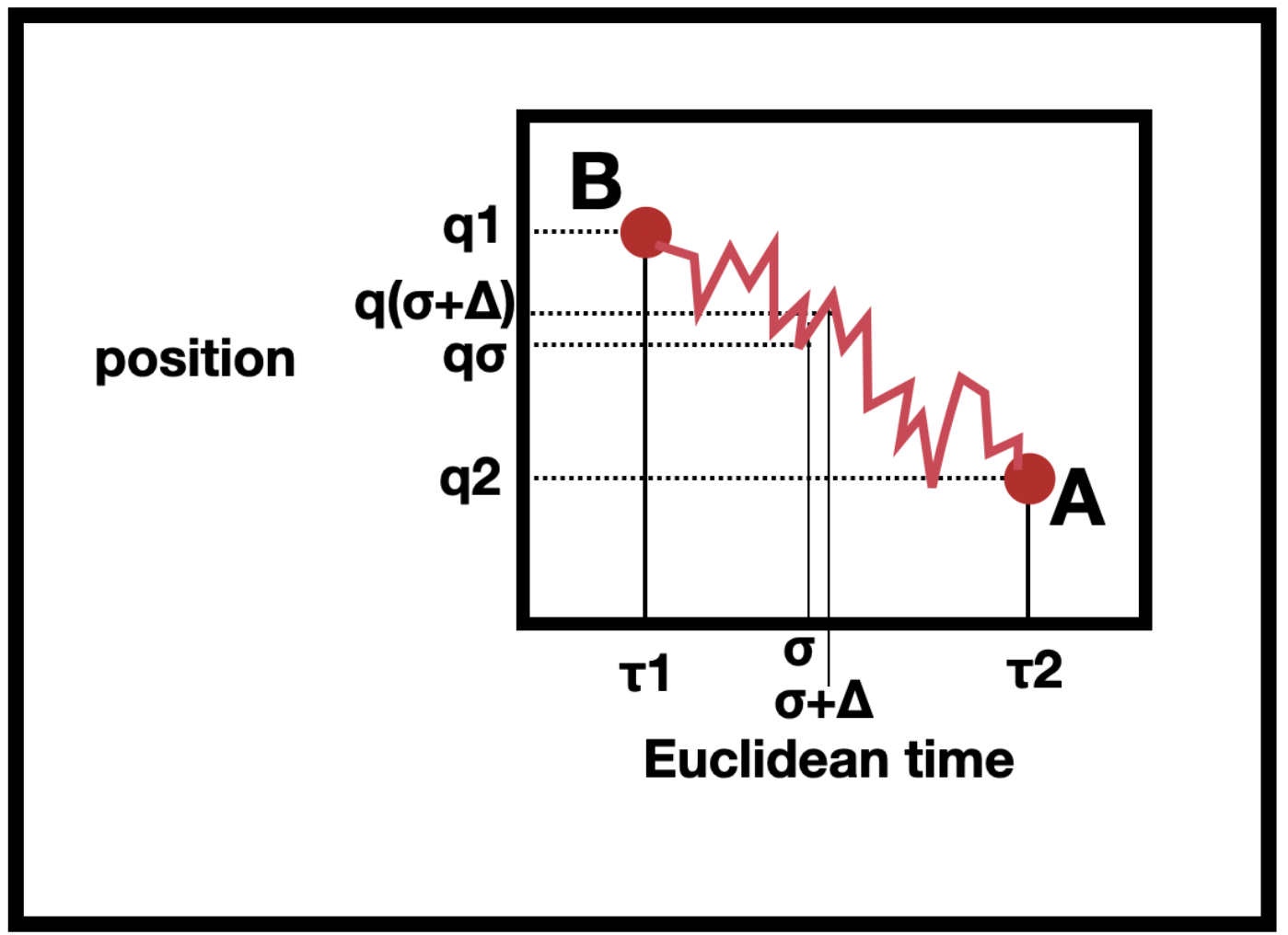

Figure 5: History of a trajectory in Euclidean time.

$$
i S(q)=i \int_{\tau_{1}}^{\tau_{2}} d s\left[\frac{m}{2}\left(\frac{d q}{d s}\right)^{2}-V\left(q_{s}\right)\right]
$$

We then replace the variable $s$ with $-i \sigma$. This results in:

$$
=i(-i) \int_{\tau_{1}}^{\tau_{2}} d \sigma\left[\frac{m}{2}\left(\frac{d q}{d \sigma}\right)^{2}-V\left(q_{\sigma}\right)\right] .
$$

So, therefore, we define the Euclidean action associated with the histories in Euclidean time:

$$
S_{E}[q]=-\int_{\tau_{1}}^{\tau_{2}} d \sigma\left[\frac{m}{2}\left(\frac{d q}{d \sigma}\right)^{2}+V\left(q_{\sigma}\right)\right] .
$$

\section{$\bigwedge$ Lagrangian in Euclidean time}

which is the Euclidean action associated to a "history" in Euclidean time $\sigma$. As noted earlier, each "history" represents a possible path or trajectory from B to A. We sum over all possible histories

$$
\begin{aligned}
\mathbf{R}-\text { Propagator }=\mathbf{R} \text {-Kernel } & =\mathbf{K}_{E}\left(q_{2}, \tau_{2}, q_{1}, \tau_{1}\right)=\left\langle q_{2}\right|\left(U_{E}\left(\tau_{2}-\tau_{1}\right)\left|q_{1}\right\rangle=\right. \\
\text { Euclidean path integral } & =\int_{\tau_{1}}^{\tau_{2}} \mathcal{D}[q(\sigma)] \exp \left(-\frac{i}{\hbar} S_{E}(q)\right)
\end{aligned}
$$

$\bigwedge$ script $\mathcal{D}$ (i.e. integrate over all paths)

which corresponds to matrix elements of the density matrix in Euclidean time.

We postulate on the graphs that there is a trajectory from $(\mathrm{t} 1, \mathrm{q} 1)$ to $(\mathrm{t} 2, \mathrm{q} 2)$ that minimizes the action, and all the other trajectories are of higher Action.

Ravi Teja next shows that these equations are properly normalized by the variable $1 / Z$, where $\mathrm{Z}$ is a partition function of a statistical ensemble of "histories." 


\subsection{The R-propagator banishes quantum weirdness}

As we keep saying, in QED the R-propagator solves a problem that Feynman did not know how to solve. With the R-propagator particles no longer need to take EVERY pathway. They can take only one. The extraordinary accuracy of QED is accounted for by the R-propagator integrating across EVERY pathway before a particle is fired from the gun.

This banishing of quantum weirdness is something that R-propagators can do in many quantum experiments and thought experiments, which is what will occupy us in the remainder of this article. As we said before, Schrôdinger's cat is no longer a paradox if wave function collapse occurs when a particle is emitted at point A. Schródinger's cat exists iff wave function collapse occurs when a particle is detected at point B (i.e. when someone opens the lid and the superposition collapses). In our theory, the cat was dead or alive when it was exposed to cyanide. The cat was not in a superposition.

We mentioned before that our proposed R-propagator also solves a problem that John von Neumann articulated. He said that the Schrôdinger equation is deterministic, and therefore, he asked, how did the randomness get into QM? No one had an answer to that question until now. As we see in Fig. 1, it is the particle that is the source of randomness. That particles are intrinsically random is no surprise. Look at Brownian motion.

\subsection{Where these ideas came from}

The concept of an R-propagator arises out of a 50 year history of something called the Theory of Elementary Waves (Boyd 2020b). Lewis E. Little, with a PhD in physics, found quantum weirdness unacceptable. He believed it meant that something fundamental was wrong in the starting assumptions of QM. He made it his life's work to figure out what.

Over thirty years Little worked alone, talking to no one, reviewing quantum mechanics, trying to come up with better starting assumptions. That is four times as much time as Andrew Wiles was sequestered working on Fermat. Little's daytime job was on Wall Street, trading in commodities. Nights and weekends he focused on his obsession. In 1993 he stumbled on the idea that quantum waves travel in the opposite direction as quantum particles. Upon that foundation he built TEW.

He expected the physics community to applaud his achievement. He expected to get rich and famous. Instead there was almost universal disinterest or rejection. He would submit a scholarly article to a physics journal, and it would be rejected with a comment, "This is not science," or "Not appropriate for this journal." Eventually a journal (Physics Essays) agreed to publish his idea in 1996 (Little 1996). Although he was invited to speak at the Jet Propulsion Labs (Little 2000), scientific interest in his idea dwindled and petered out. The risk was that when our generation died of old age, TEW would vanish from the face of the earth.

This author is Little's cousin. He has had a huge impact the author's life. It is because of him that we chose to go to the college we did. We have been talking and arguing about many things for more than half a century. He is the most argumentative person we ever met. Once he bickered with the author for an hour about why the author refused to bicker with him.

He taught the author TEW, then assigned us the task of developing a mathematics for TEW. Unlike Little, the author has a degree in mathematics (Brown University, 1965). The author spent the years from 2010 till now speaking about TEW at physics conventions, publishing scholarly articles in refereed journals of physics and math, and making YouTube videos (Boyd 2010-2020).

In 2013 Little and the author had a fight leading to what amounts to a "divorce." In terms of ownership of TEW as a new idea the author was committed to the idea of public domain, whereas Little was committed to private property. They have not communicated since (Boyd 2020b).

The author continues to build a mathematics for TEW. That is where this article comes from. The question is whether "TEW" should be renamed "Theory of R-Propagators" (TRP)?

\section{The mathematics of Nature is simplified if we adopt R-propagators}

In this section we will demonstrate with data from published experiments that experiments change their meaning if we assume there are R-propagators instead of propagators. It is like looking at a mirror image of the world you thought you knew. The QM view of the quantum world is like Lewis Carroll's Through the Looking Glass (1871). In that book Alice looks in a mirror (a "looking glass"), then pops through the mirror, into the mirror-image world. She discovers that the mirror image world is bizarre beyond imagination. Propagators and R-propagators are mirror images of one another. Only one of them is found in Nature. If you assume that Nature uses propagators, then you are in a mirror-image world which is bizarre beyond imagination. Yet the equations of QM are identical in the mirror image world as in the real world. 


\subsection{A neutron interferometer experiment demonstrates the existence of R-propagators}

A research team founded by Helmut Rauch did the basic research that created the field of neutron interferometry. The team was and is highly respected. The publication which we will focus on is written by Helmut Kaiser, Russell Clothier, Samuel Werner, Helmut Rauch, and H. Wölwitsch and was published in Physical Review A in 1992. The authors said that their results could not be explained by QM. Indeed the results conform to the predictions of R-propagators, and contradict the predictions of propagators.

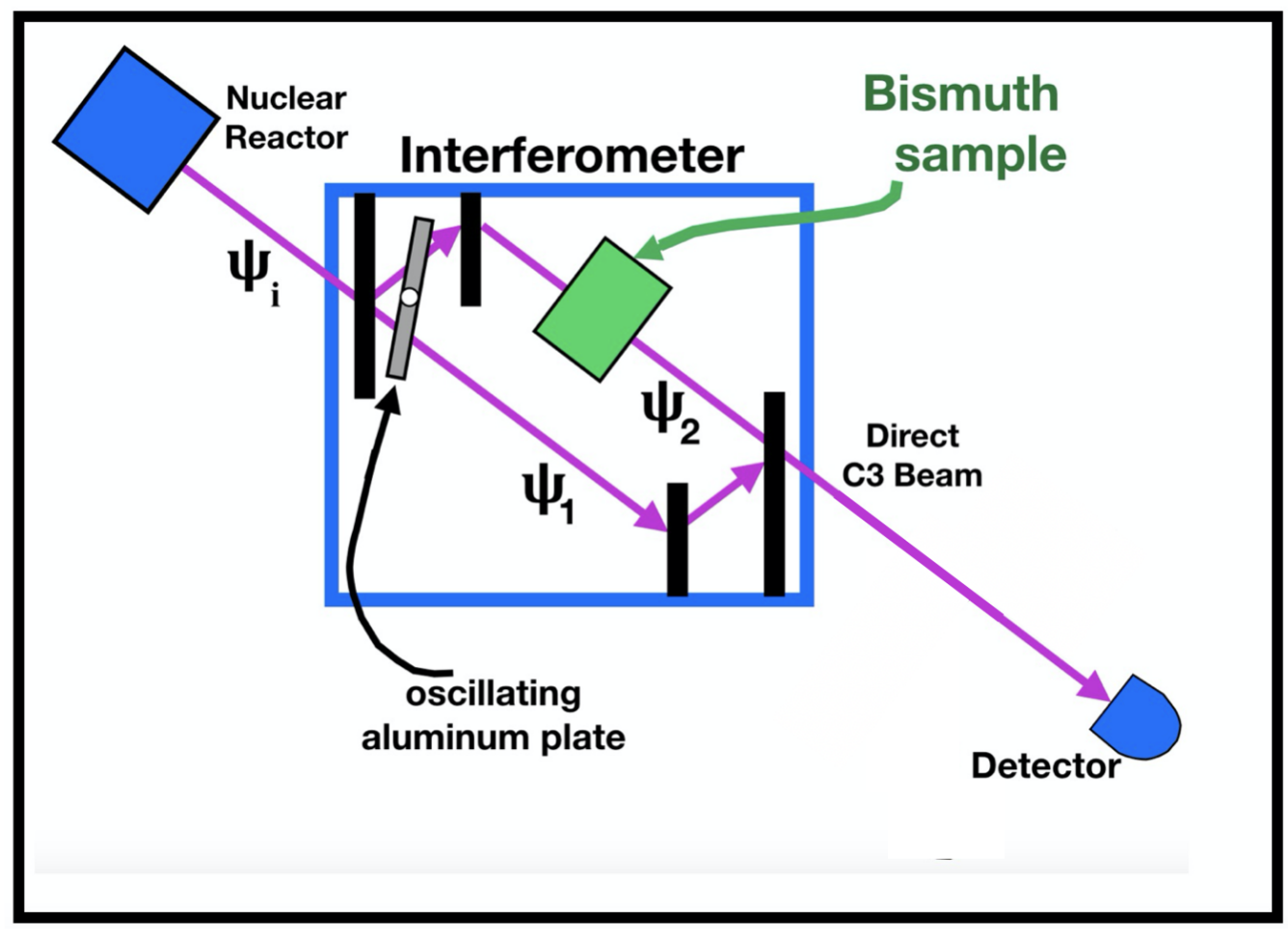

Figure 6: Neutron Interferometer experiment.

Fig. 6 shows the equipment they used for a series of experiments. Bismuth, the 83rd element, is a metal that slows neutron beams. The series of experiments used increasing amounts of Bismuth, from zero to $20 \mathrm{~mm}$ thick. They machined samples of Bismuth to different thicknesses. It is a soft metal and difficult to work with in a machine shop. A sliver of soft metal $5 \mathrm{~mm}$ thick is extremely delicate, and demanded utmost attention to insert that delicate sliver into the tiny interferometer.

The detector is black to thermal neutrons, and is a 0.5 inch, cylindrical, 20-atm ${ }^{3} \mathrm{He}$ detector mounted on a neutronshielding $B_{4} C$-epoxy cassette with a $1 \mathrm{X} 1$ inch collimated opening. This made the probability of detection constant in a horizontal plane.

An incident neutron beam $\Psi_{i}$ enters the interferometer where it bounces off various silicon blades (shown in black). When the two beams, $\Psi_{1} \& \Psi_{2}$ separate, there is an oscillating aluminum plate that causes a phase difference between the two beams, so that when they are later re-combined there is wave interference, evident in there being a sinusoidal curve. As more and more Bismuth is added, the upper wave packet $\Psi_{2}$ slows relative to the lower wave packet $\Psi_{1}$ so they don't overlap so well when they rejoin to leave the interferometer. As the amount of Bismuth increases, the sinusoidal wave (i.e. interference) flattens out and eventually becomes a flat line, indicating no interference. This is evident in the middle column of Table 2, where the sinusoidal curve falls from $100 \%$ to $02.9 \%$, which is background noise or static.

They then repeated exactly the same series of experiments, with one tiny difference. They inserted a "Nearly Perfect Analyzer Crystal" (shown in green, lower right) into the exit beam. The research team had to decide what analyzer crystals to use, and what Bragg angles. Here we will report data gathered from a "nearly perfect" (NP) silicon crystal: $\Delta \theta=0.02^{\circ}$ full width at half maximum $(\mathrm{FWHM}), \eta_{A}=0.0035 \mathrm{rad}$. They used this NP Analyzer Crystal in the (111) antiparallel configuration. With an incoming neutron beam of $\lambda=2.35$ the Bragg angle that they use is $\theta_{A}=-22.0^{\circ}$. They used the same detector, but it was rotated to a new position (Fig. 8). 


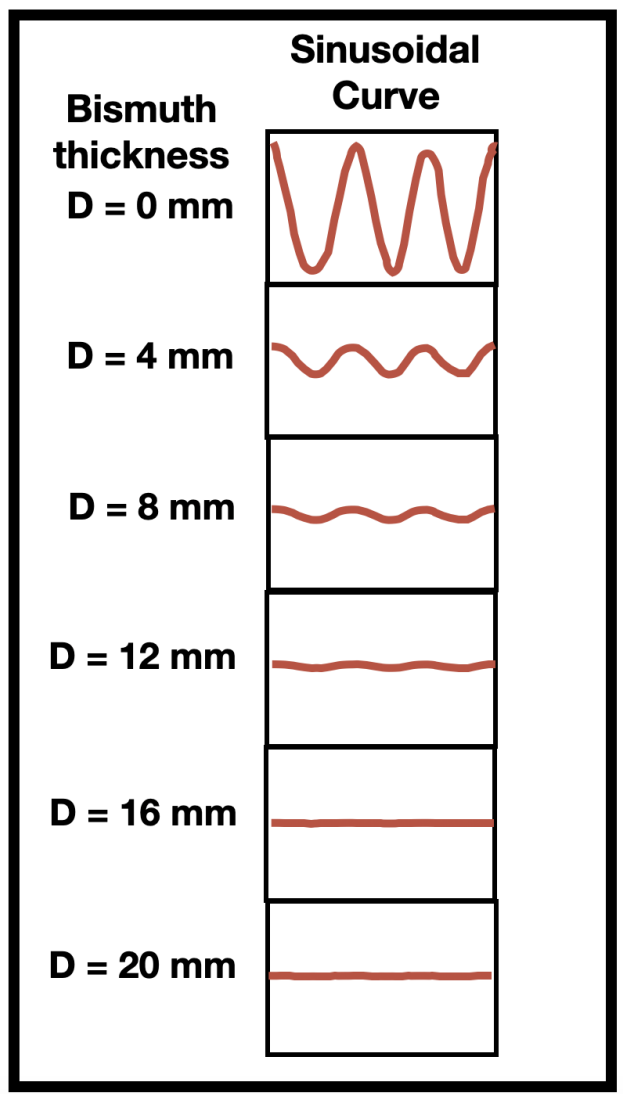

Figure 7: With an increasing sample of Bismuth, measured in millimeters, the interference seen by the detector dies out.

Table 2

Height of sinusoidal waves without \& with Analyzer Crystal

\begin{tabular}{|r|r|r|r|}
\hline$\#$ & Bismuth width & No Analyzer Crystal & With Analyzer Crystal \\
\hline 1. & $0.00 \mathrm{~mm}$ & 100.0 & \multicolumn{1}{c|}{$\begin{array}{c}\text { \% } \\
\end{array}$} \\
\hline 2. & $2.09 \mathrm{~mm}$ & $88.1 \pm 1.3 \%$ & $97.1 \pm 5.1 \%$ \\
\hline 3. & $4.01 \mathrm{~mm}$ & $57.3 \pm 1.0 \%$ & $99.0 \pm 4.8 \%$ \\
\hline 4. & $12.26 \mathrm{~mm}$ & $8.0 \pm 0.8 \%$ & $89.6 \pm 4.4 \%$ \\
\hline 5. & $16.15 \mathrm{~mm}$ & $1.8 \pm 0.8 \%$ & $86.0 \pm 4.8 \%$ \\
\hline 6. & $20.08 \mathrm{~mm}$ & $2.9 \pm 0.6 \%$ & $95.2 \pm 5.2 \%$ \\
\hline
\end{tabular}

The effect of this Analyzer Crystal (Fig. 9) is to focus the beam: decrease the scatter of wavelengths and increase the Gaussian in the center. The expected effect would be to cause the beam to penetrate more intensely into the detector. But the Analyzer Crystal was outside and downstream from the interferometer, and therefore there was no way in which it could have an impact on the interference inside the interferometer.

The researchers were astonished with their data, shown in the right-hand column of Table 2 . The data showed that the insertion of an Analyzer Crystal totally restored all the interference upstream, inside the interferometer. They were flabbergasted. For example, look at the bottom row of Table 2. With a maximum sample of Bismuth (20.08 $\mathrm{mm})$ the height of the sinusoidal curve was $95.2 \%$ of what it would be if there were no Bismuth (top and bottom numbers on the right-hand column.

The researchers said QM cannot explain this, which contradicts the dogma that "QM can explain ALL experimental data." They proposed various explanations that made no sense, such as the idea that the Analyzer Crystal managed to conjure up a "coherent" beam of neutrons out of an incoherent beam.

Comparing this experiment to the Feynman path integral experiments cited above, this one is much simpler. There is only one trajectory, which is split into two $\left(\Psi_{1}\right.$ and $\left.\Psi_{2}\right)$ inside the interferometer, then rejoined to leave the interferometer 


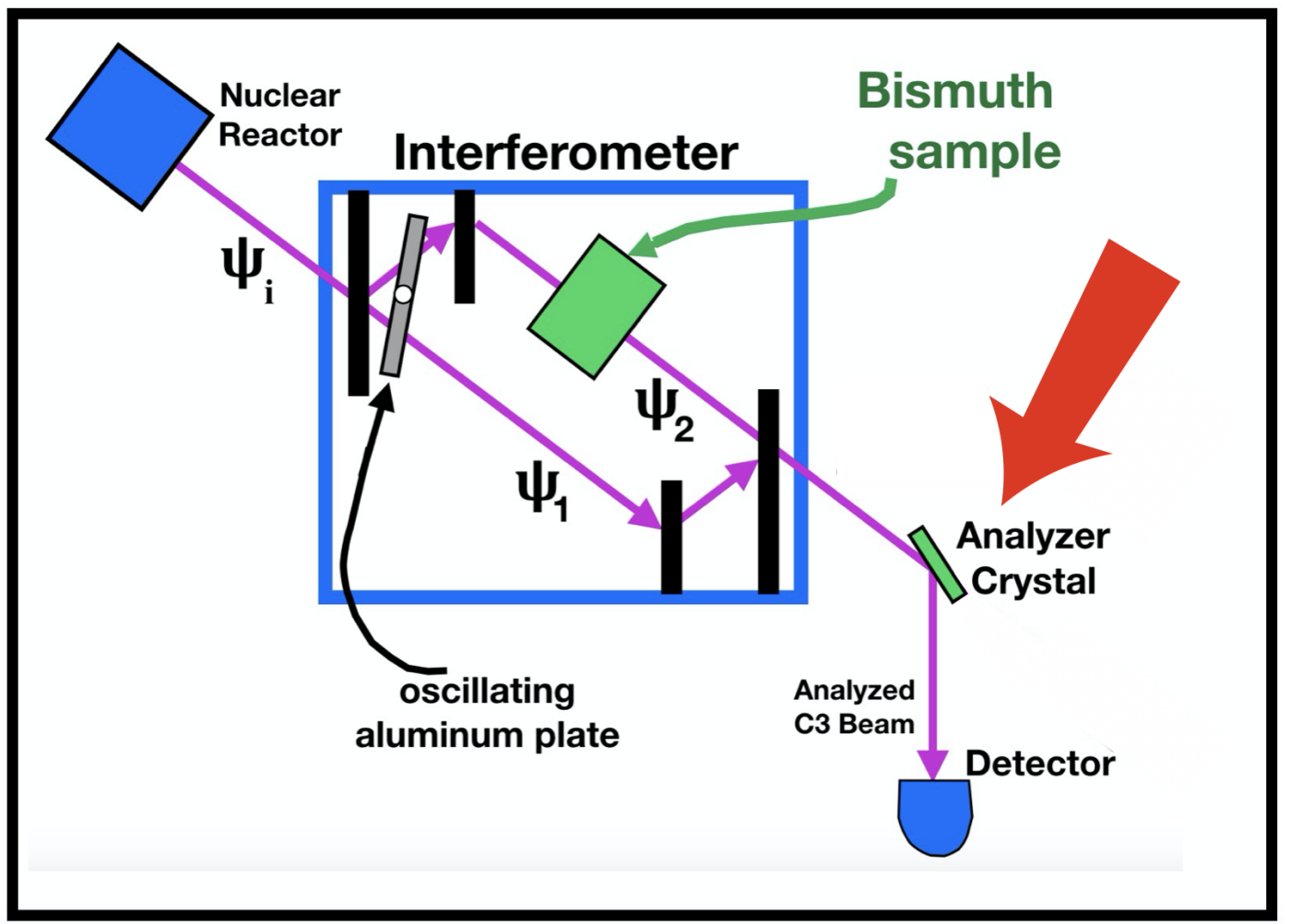

Figure 8: The research team then swung detector C3 down (lower right) and inserted an "Analyzer Crystal" of pressed silicon that had the effect of focusing the neutron beam so it should penetrate better into the detector. But this was not expected to have any effect on the interference that previously occurred upstream inside the interferometer.

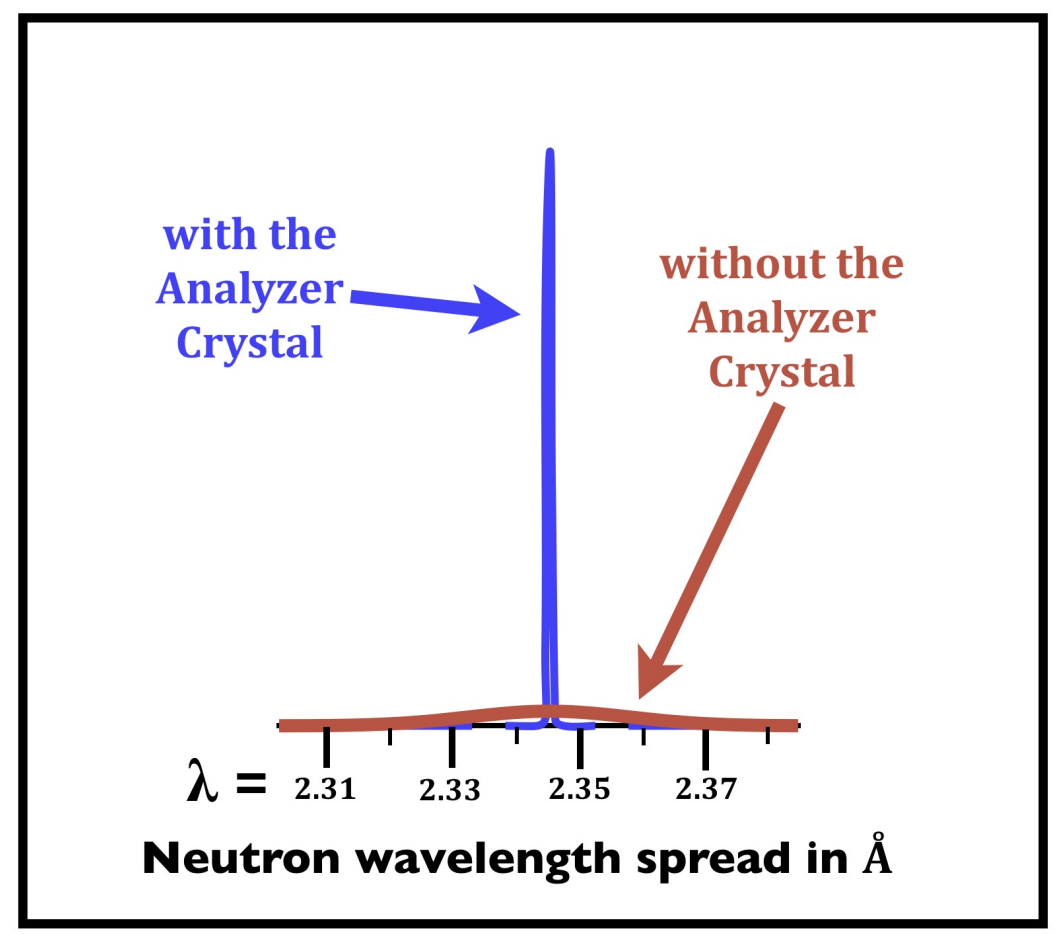

Figure 9: Gaussian curves for the presence (blue) versus absence (red) of an Analyzer Crystal. 


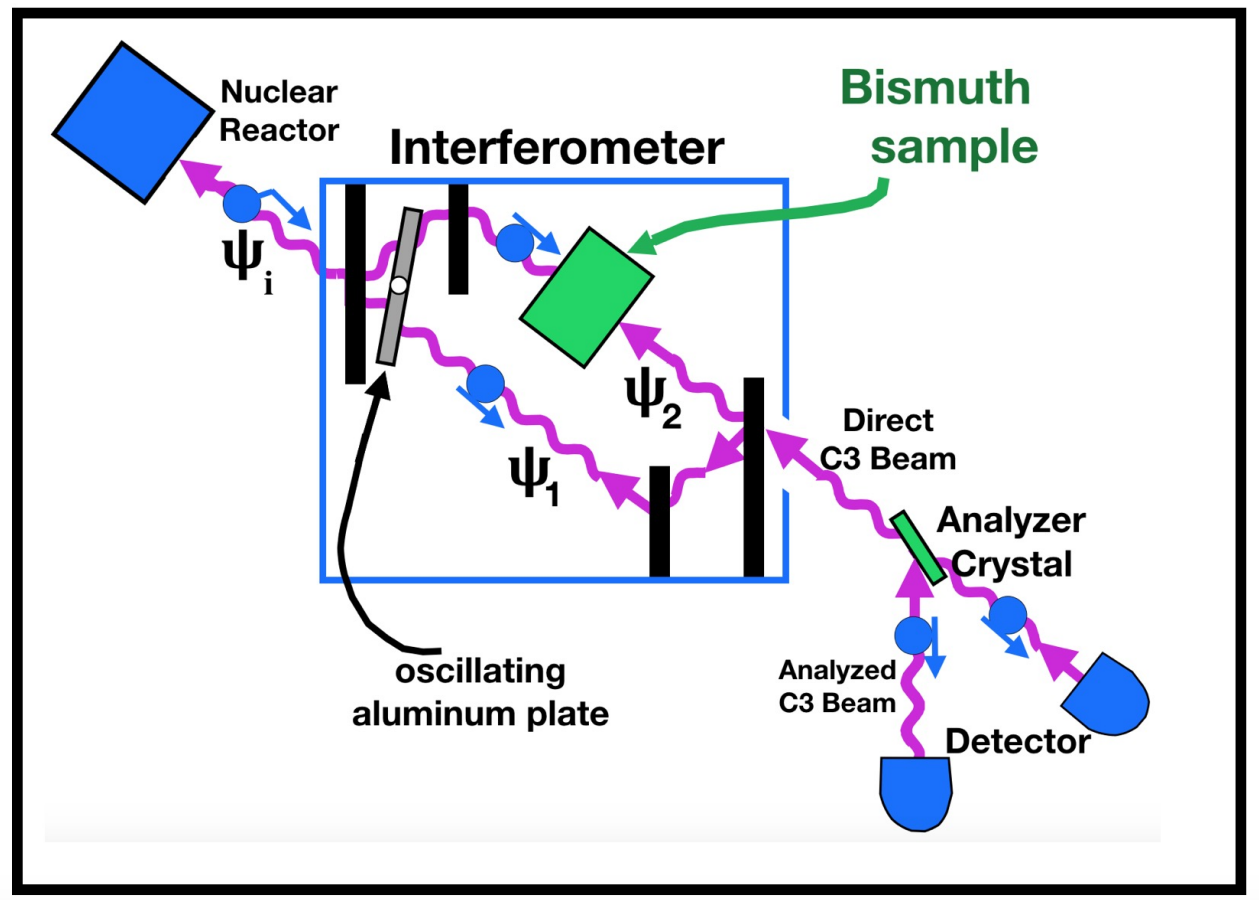

Figure 10: Our interpretation of the neutron Interferometer experiment. The purple waves represent R-propagators traveling centripetally toward the nuclear reactor, and neutrons follow them backwards, centrifugally away from the nuclear reactor.

and hit the detector. The assumption of the researchers is that the propagators started at the Nuclear Reactor in the upper left, followed the purple arrows and struck the detectors, and the neutrons followed the projectors.

If you stop and think logically, we have an experiment in which the presence or absence of interference inside the interferometer is determined by the presence or absence of an Analyzer Crystal in the lower right corner of the experiment, in front of the detector, then the Analyzer Crystal must be UPSTREAM from the interference.

The experiment can only be explained if there are R-propagators starting at the detector, going backwards through the equipment, and entering the nuclear reactor. From time to time a neutron from a disintegrating atom would follow the R-propagators backwards. Our model is diagrammed in Fig. 10, with the R-propagators symbolized by purple waves going from lower right up into the nuclear reactor (upper left) and blue neutrons moving in the opposite direction.

With no Analyzer Crystal a wave packet had a coherence length of $\Delta X=86.2$ Angstroms. A sample of $20 \mathrm{~mm}$ of Bismuth would delay the wave packet by 435 Angstroms. So it is easy to see why all interference died out as more and more Bismuth was added. However with the addition of an NP Analyzer Crystal, the $\Delta X$ coherence length increases to 3450 Angstroms. So it is easy to see why waves penetrating backwards through the interferometer would easily pass through a $20 \mathrm{~mm}$ block of Bismuth, as if it were invisible.

\subsection{The double slit experiment with NO wave particle duality}

Usually when someone speaks of wave particle duality, they are thinking primarily about the double slit experiment. The theory claims that a single particle leaves the gun, and must go through both slits, as evident from the wave interference pattern on the target screen.

The problem is that Einstein proved that picture is WRONG. He proved it cannot possibly be a correct picture. But, alas, Einstein didn't listen to himself, and did not draw the obvious conclusion that wave particle duality is wrong. We have a wave particle duality problem today because Einstein was indecisive, and failed to make up his mind.

Einstein's criticism of the conventional model is simple: Once a dot appears anywhere on the target screen it is necessary for the ENTIRE cloud of probability amplitudes everywhere to vanish, faster than the speed of light. If it did not all vanish instantaneously, then some other part of the cloud could produce a second dot, which would be impossible since only one particle left the gun. Another way to state it is: once a dot appears, wave function collapse has occurred, so all the probability density is located at that dot, and there is no excess probability density available for the cloud. Therefore the cloud must instantaneously vanish, faster than the speed of light. 

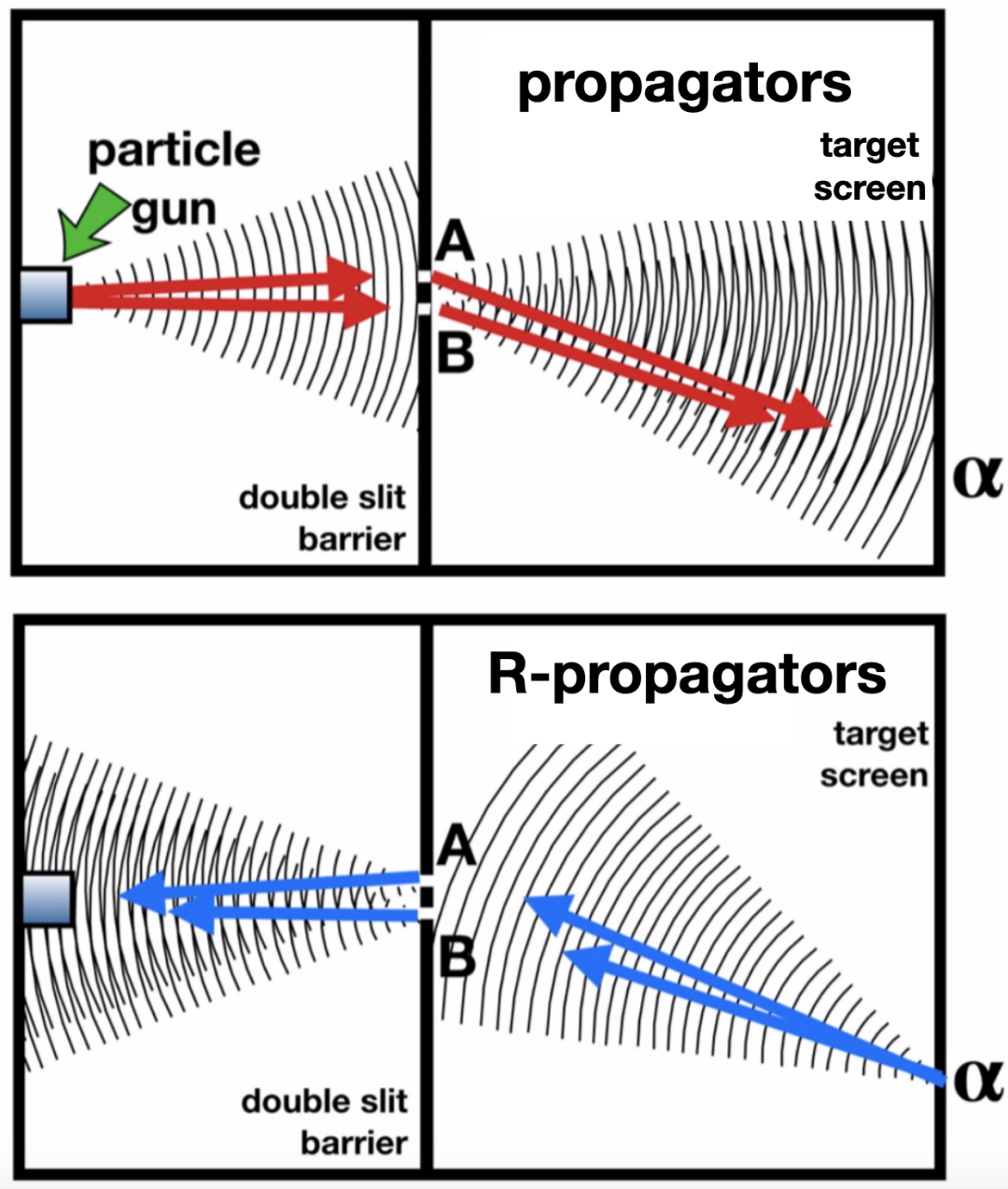

Figure 11: Two divergent theories for how a double slit experiment makes a dot at point $\alpha$ on the target screen.

No one ever answered Einstein's complaint. No one ever proposed any mechanism by which the entire cloud of probability amplitudes would instantaneously vanish, faster than the speed of light. This means that Einstein's argument destroys the conventional view of the double slit experiment.

The conventional wisdom taught in all the textbooks and all over the internet, is WRONG! Einstein demolished it 100 $\%$. Nevertheless it continues to be taught everywhere as if it were true.

Therefore the double slit experiment cannot be used as evidence of wave particle duality. In our multiple articles on this subject we have demonstrated that the other experiments about wave particle duality, such as the Davisson-Germer experiments (Davisson \& Germer1927; Davisson 1928a, 1928b, Boyd 2013b, 2019b), prove that waves and particles interact, but not that they are identical. Even de Broglie thought that way (Freire 2003).

The experiments of Pfleegor and Mandel $(1967,1968)$ disprove wave particle duality. The experiment by Kaiser, Clothier, Werner, et. al. (1992) proves that wave particle duality cannot be true.

Fig. 11 offers an unconventional explanation of the double slit experiment. Since both the conventional explanation (Fig. 12 top) and our explanation (Fig. 11 bottom) produce identical results, and since Einstein disproved the conventional explanation but his critique does not apply to our explanation, therefore our explanation is the ONLY explanation that works.

We claim that R-propagators flow out of every point on the target screen, in every direction. We are going to picture them going in straight lines. We differ from Feynman because he never liked straight lines.

The bottom of Fig. 11 shows that the R-propagators from point $\alpha$ strike slit A at a different time than they strike slit $\mathrm{B}$, unless $\alpha$ is in the center of the screen. They penetrate those slits backwards with phase $\theta_{A}$ and $\theta_{B}$. That determines a phase difference $\left(\theta_{B}-\theta_{A}\right)$, which is the number we want to focus on. That phase difference persists as 
the R-propagators from slits A and B converge on the particle gun. The phase difference $\left(\theta_{B}-\theta_{A}\right)$ determines whether wave interference at the gun is constructive, intermediate, or destructive. That determines the amplitude and intensity with which the R-propagator from point $\alpha$ presents itself to the particle about to be fired from the gun.

The particle looks out and sees a zillion incident R-propagators converging on it, each with its own amplitude. The word "zillion" refers to a large finite integer. The particle randomly chooses one, and only one R-propagator to respond to.

The particle then follows its R-propagator with a probability of one, through one and only one of the slits (it doesn't matter which slit) and inevitably makes a dot at point $\alpha$ on the target screen.

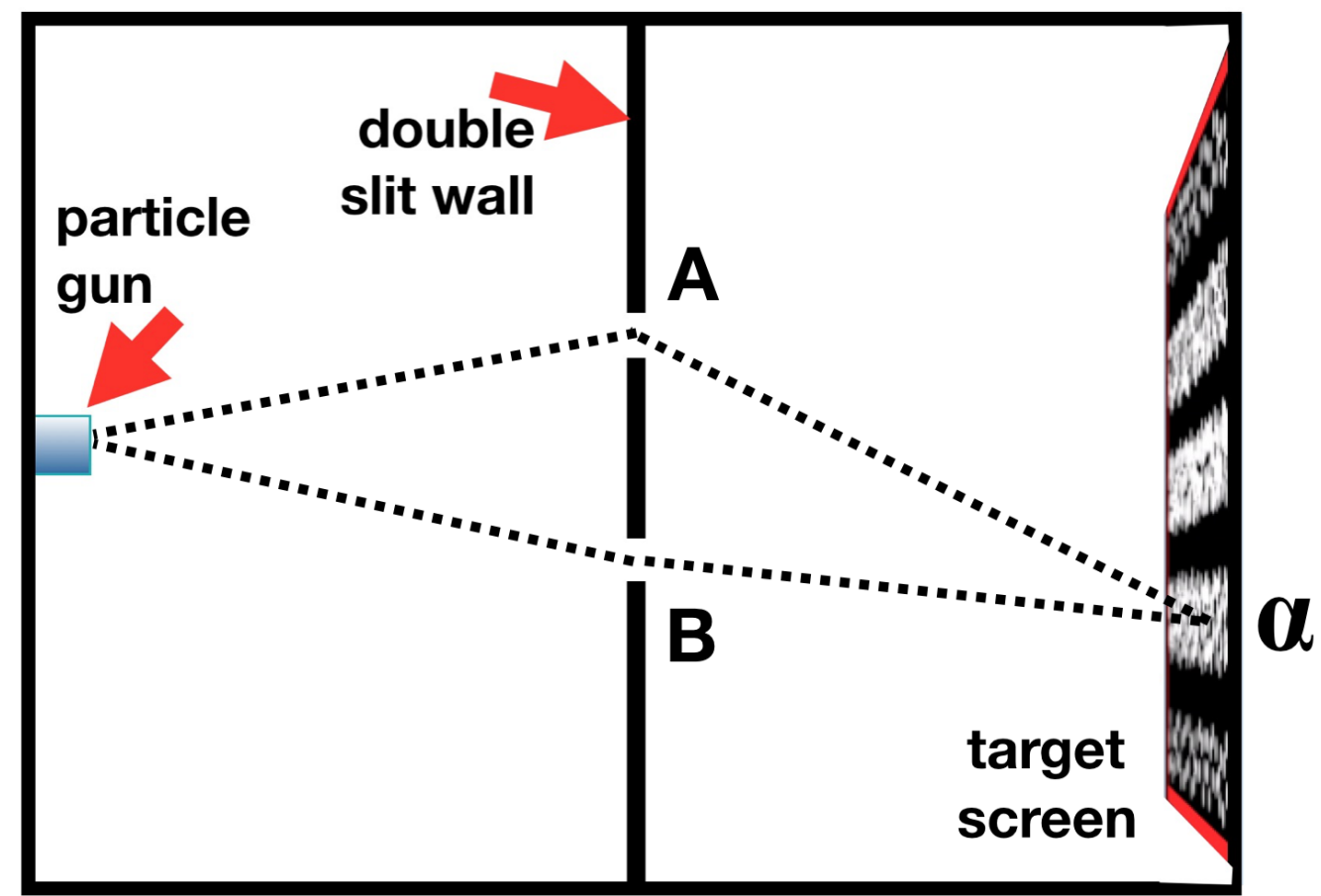

Figure 12: This diagram explains why propagators and R-propagators produce the same results.

Fig. 12 demonstrates why the pattern on the target screen is the same, whether you think of propagators traveling from left to right, or R-propagators from right to left. In Fig. 12 there are two pathways (dotted lines): from the gun to slit A to point $\alpha$ on the target screen; and from the gun to slit B to point $\alpha$. If we measure them both, subtract one from the other, then divide by the wavelength $(\lambda)$, the modulo divided by $2 \pi$ is the phase difference. Our point is that the phase difference $\left(\theta_{B}-\theta_{A}\right)$ is the same whether the propagator move from left to right, or the R-propagator moves right to left. The phase difference produces the wave pattern in the final dataset.

We have now proved that the pattern on the target screen can equally well be explained by either propagators or R-propagators. Einstein demolished the propagator explanation of the double slit experiment. Therefore our theory of R-propagators is the ONLY possible explanation of how a double slit experiment works.

Einstein's critique of the conventional model does not apply to our R-propagator model because after a dot appears on the target screen there is no cloud of probability amplitudes that needs to vanish.

\subsubsection{Why observing a slit destroys the interference pattern on the target screen}

If a lamp and detector are inserted into a double slit experiment in order to observe which slit the particle uses, the interference pattern on the target screen vanishes. Our theory says that the energy emitted by the lamp, no matter how infinitesimal, is still infinitely more energy than the R-propagator in that neighborhood, which conveys zero energy. The effect is to destroy the superposition additivity of the R-propagator going backwards through slit A and the R-propagator going backwards through slit B. The two R-propagators no longer interfere as they impinge on the gun.

What does the pattern on the target screen signify in a double slit experiment? According to our theory that pattern is a picture of the interference of R-propagators impinging on the particle gun. Given what we said in the previous paragraph, there is no wave interference incident to the gun if a detector is observing one of the slits. Therefore the target screen displays no wave interference. 


\subsection{A proposed experiment testing propagators versus $\mathbf{R}$-propagators}

So far we have presented two experiments for which the data are consistent with R-propagators, but incompatible with the propagator theory, namely

- A neutron interferometer experiment, and

- The double slit experiment.

We will now design of a new experiment, never conducted, that would produce different results if R-propagators are what Nature uses, than if Nature uses propagators.

The experiment is shown in Fig. 13. Particles are fired one at a time. The timing for this experiment must be precise down to the nanosecond. At that instant $(t=0)$ when the gun fires a particle, a powerful laser closes the right hand slit. Therefore we have a double slit experiment when $t<0$ but a one slit experiment when $t>0$. If Nature uses propagators then this is simply a single slit experiment, so the result should be that a single vertical line is seen on the target screen, because of the particles going through the left slit.

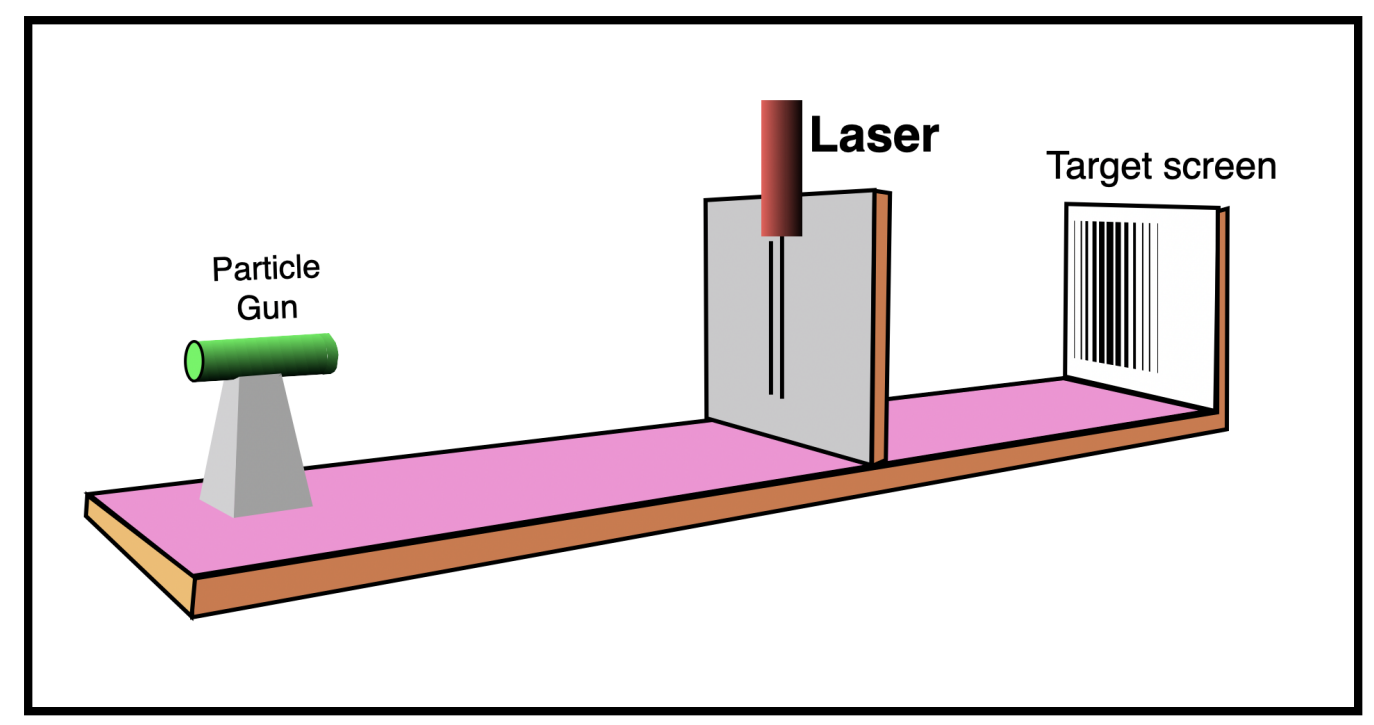

Figure 13: A proposed experiment to discover whether Nature uses propagators or R-propagators. Particles are fired one at a time from the gun at time $t=0$. At that nanosecond $(t=0)$ a laser closes the right-hand slit. If Nature uses propagators then no interference pattern should appear on the target screen, simply one vertical line. If Nature uses R-propagators then we should see the pattern shown here: an interference fringe pattern skewed to the left.

But if Nature uses R-propagators the result will be a skewed wave fringe pattern as shown in the Figure. Why? During the time $t<0 \quad$ R-propagators were penetrating through both slits and impinging on the gun, where there was wave interference. In our theory the target screen accurately portrays whatever wave interference is occuring incident to the gun. Therefore the pattern of particles leaving the gun will reflect the wave interference that has already occurred at $t \leq 0$. However half the particles are unable to get past the barrier, because the right slit is now closed.

Because propagators and R-propagators produce identical results in almost all experiments, you need a moving part in order to draw a distinction. The vertical laser provides such a moving part.

\subsection{A thought experiment from John A. Wheeler demolished by using R-propagators}

An article was published in Science in 2007 that changes its meaning when we use the concept of R-propagators. The article, however, never mentions "propagators" (Jacques, Wu, Grosshans, et.al. 2007). It reported an experiment focused on an idea of John Archibald Wheeler about quantum weirdness. The experiment allegedly "proved" that the quantum world is weird.

Wheeler pondered the idea of wave particle duality. If we look for a wave then we see a wave, but if we look for a particle then we see a particle. So he dreamed up a way to force the particle to make a decision at the front door of an interferometer as to whether it was a wave or a particle.

Fig. 14 shows two ways that an interferometer can be arranged: open or closed. The open configuration is used to detect particles striking detectors D1 or D2. The closed arrangement looks for wave interference. The doctrine of wave 


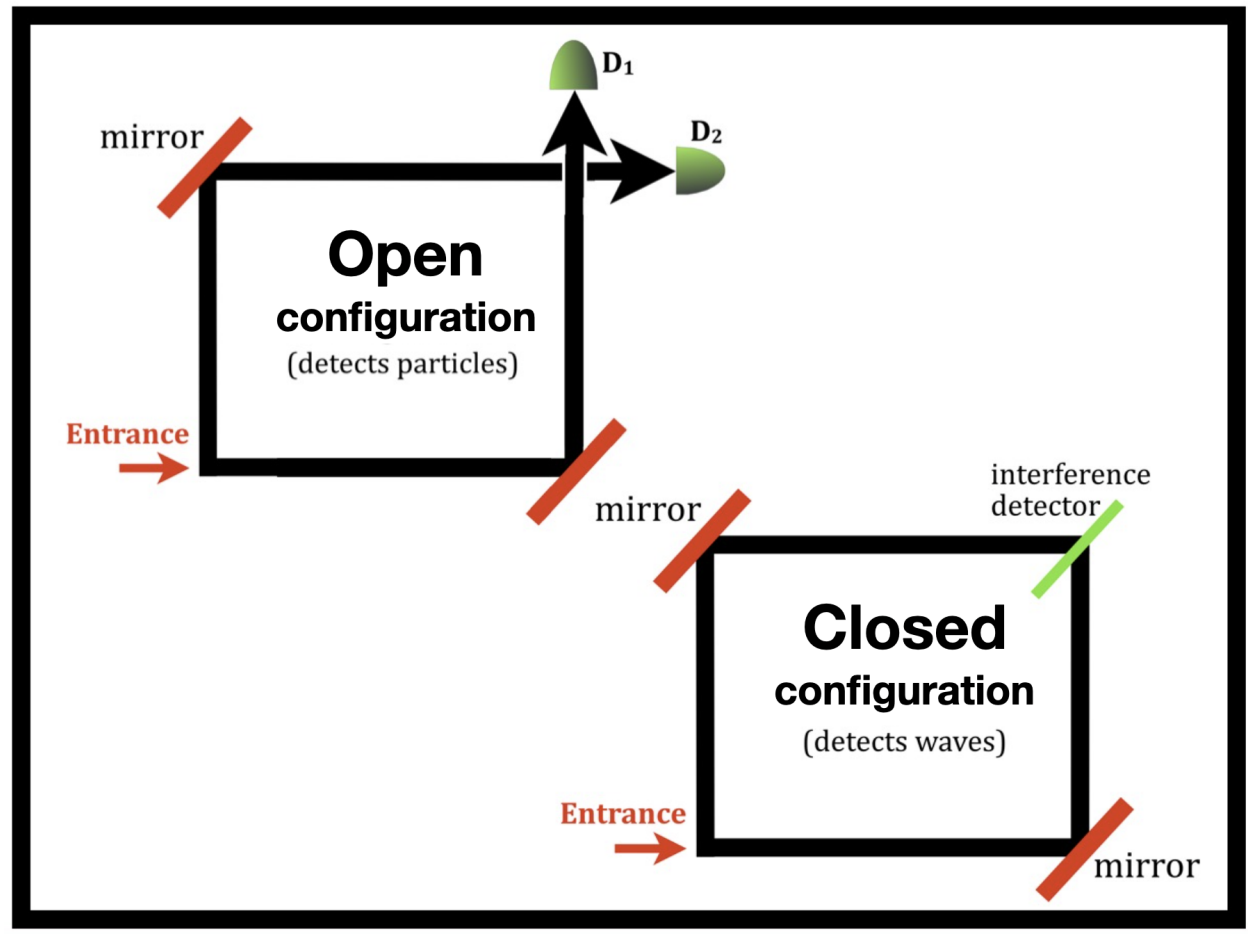

Figure 14: Wheeler's idea about two arrangements of an interferometer. Upper left, an open configuration tests for a particle traveling across one of the arms of the apparatus and causing either detector D1 or D2 to "click." Lower right, a closed configuration looks for interference caused by a wave crossing both arms of the interferometer simultaneously and causing interference at the intersectiont.

particle duality proposes that if you use the open configuration then you will see a particle, but if you use the closed configuration then you will see a wave. So if you built an experiment in which the testing equipment could be switched from open to closed configuration and back in a few nanoseconds, then you might catch the quantum of energy trying to make up its mind whether to be a wave or a particle. Somehow, Wheeler reasoned, the arrangement of the testing equipment is transmitted to the particle, warning it whether it will be tested as a wave or a particle.

The question is whether this transmission of information could happen backwards in time, Wheeler thought. So what would happen, asked Wheeler, if we built equipment where a quantum of energy was input through the front door (lower left of the interferometer) where it would be required to decide whether it would be a particle (i.e. crossing only one of the two paths) or a wave (i.e. crossing both paths simultaneously). Then, after the quantum has made an irreversible decision, we make a decision about how to arrange our testing equipment. Would we then find that the particle conformed to our equipment, or not? Would we find that when we look for a wave we always see a wave, and if we look for a particle we always see a particle?

If an experiment did find that, then we would prove backwards in time transmission of information, from the detectors to the quantum midway in its trip across the interferometer.

A research team had already built equipment that could be adapted to answer Wheeler's question (long after Wheeler had died) (Jacques, Wu, Toury, ett. al. 2005). so they adapted their equipment and conducted empirical research that was reported in the leading journal Science, confirming Wheeler's prediction: if you look for a wave you will see a wave, if you look for a particle you will see a particle. That empirical data is interpreted to mean that time can go backwards in the quantum world. In other words an effect can precede its cause.

However, as we are about to show, the meaning of the same experiment changes if we postulated R-propagators.

\subsubsection{Equipment used in the experiment}

Fig. 15 shows the Mach Zehnder interferometer used by Jacques et al. That interferometer was 48 meters wide (half a football field), which caused the photon to take a long time (160 ns) crossing it, which gave the researchers time to have a random number generator (which took $40 \mathrm{ns)}$ ) to determine whether the equipment would subsequently test the quantum as a wave (closed configuration) or as a particle (open configuration). The switch that determined which way they were going to test the quantum was an Electrical Optical Modulator (EOM). What Jacques et. al. found was 


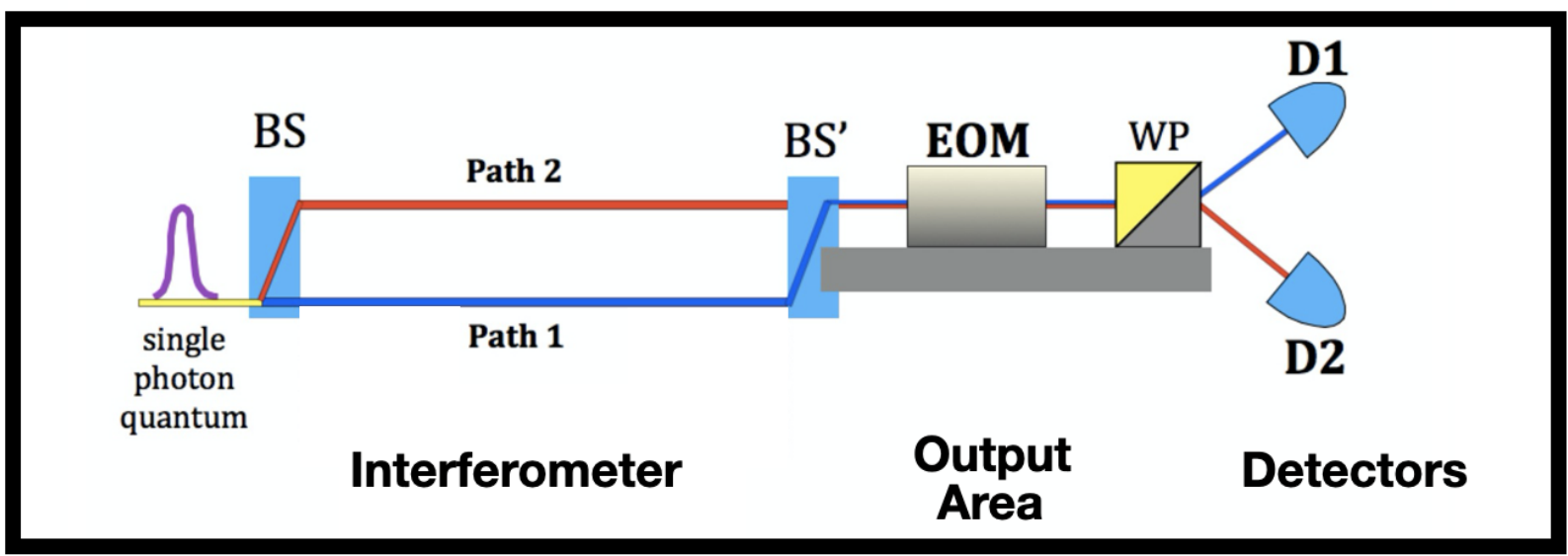

Figure 15: The Mach-Zehnder interferometer used by Jacques et. al. is as wide as half a football field. BS and BS' are $\mathrm{YVO}_{4}$ beam splitter and reverse beam splitter. The lower path (Path 1) has a horizontal polarization; the upper path has a vertical polarization. Paths 1 and 2 are separated by $4 \mathrm{~mm}$. EOM is Electrical Optical Modulator, WP is a Wollaston prism, and D1 and D2 are silicon avalanche photodiode detectors 1 and 2. After a quantum of energy enters the interferometer (lower left) and makes its decision to cross as a wave (on both paths) or as a particle (on only one path), a random number generator (not shown) causes the EOM to determine the angle of polarization, which determines how the results are measured.

that when they looked for a wave they saw a wave and when they looked for a particle they saw a particle. They declare that was "proof" that Wheeler's hypothesis was correct. Proof that the quantum world is weird.

Figs. 15 through 17 describe the equipment used by Jacques et al in the Wheeler gedanken experiment. A phase shift $\phi$ was implemented between the two pathways by having the second BS' reverse beam splitter rock back and forth. Therefore if the quantum was crossing the interferometer as a wave, there would be a phase difference between the two paths, and therefore interference inside the WP prism. This would register at the detectors (D1 and D2) as $Y= \pm \cos ^{2} \phi$.

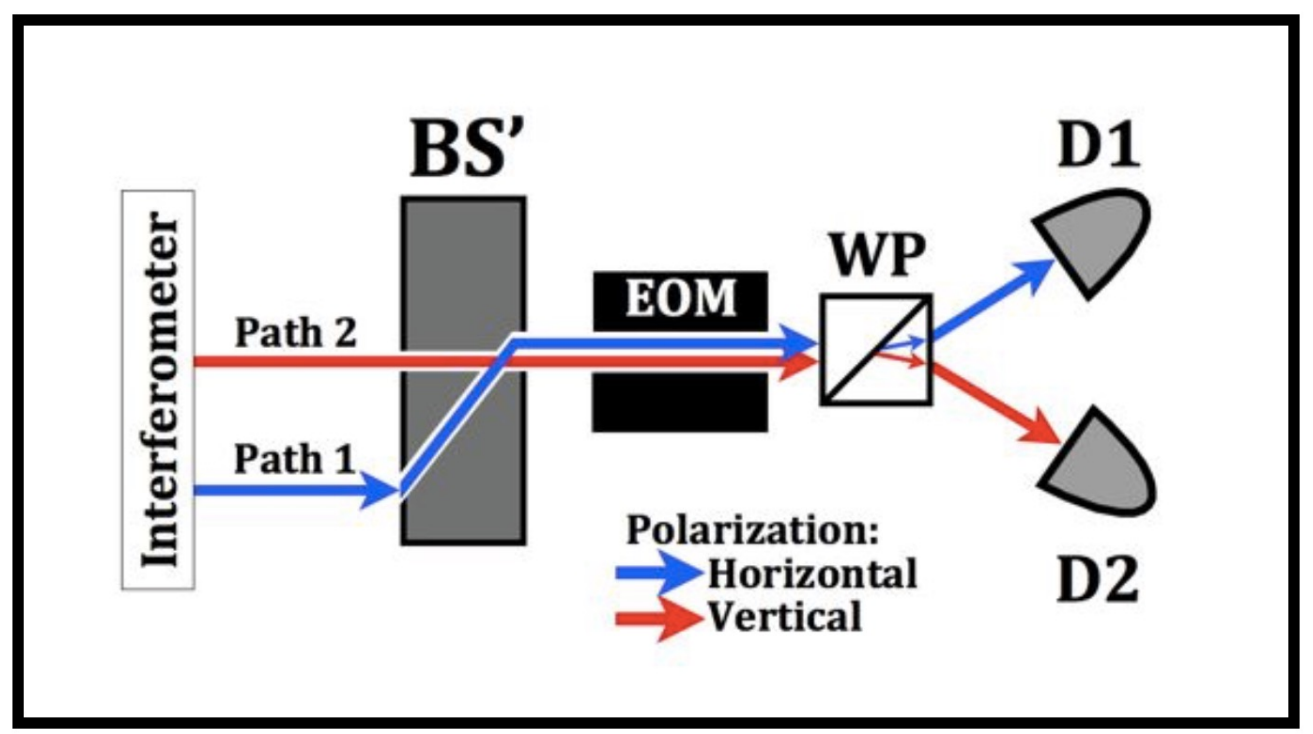

Figure 16: This shows the output area of the Jacques experiment. In the second beam splitter BS' the horizontal and vertically polarized photons are re-combined. That device (BS') rocks back and forth so as to create a phase shift and interference of $\Psi_{1}$ and $\Psi_{2}$. If the EOM is "OFF" it acts as if it is not present. The photons then enter the Wollaston prism where they are assigned to one detector or the other, depending on their polarization.

In conclusion Jacques et al. believe the graphs in Fig. 18 prove that Wheeler's hypothesis was correct. The random choice in the output area whether to test the quantum as a wave (EOM is "ON") or as a particle (EOM is "OFF") has 


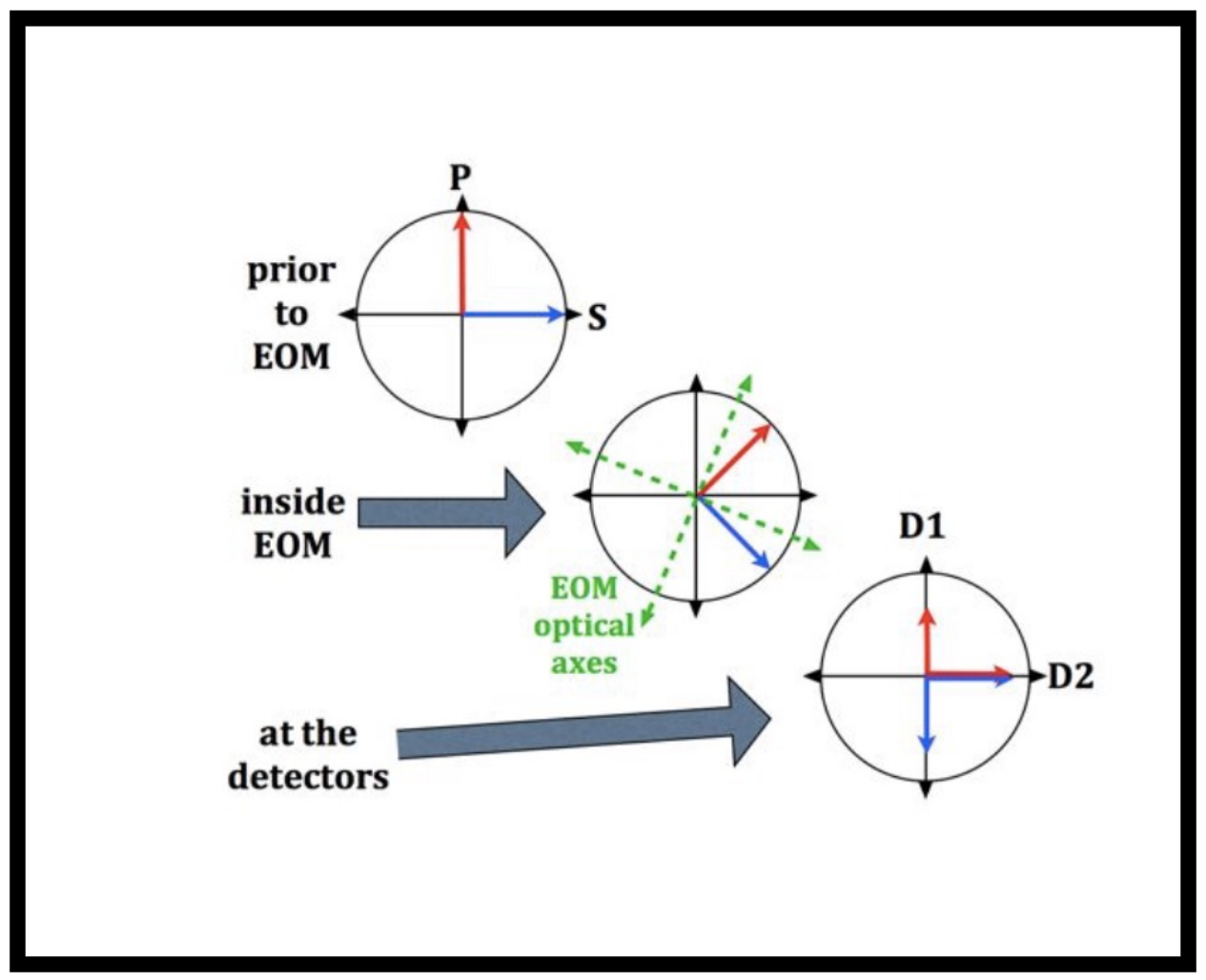

Figure 17: How the EOM affects angle of polarization. If the EOM is "OFF" it acts as if it is not present. If it is "ON" it rotates the polarization by $\pi / 4$. At the detectors, D1 gets all horizontally polarized photons, D2 gets vertically polarized, but if the axis has been rotated by $\pi / 4$ inside the EOM, the photons have interference inside the WP and have $\mathrm{a} \pm \cos ^{2} \phi$ chance of being assigned to D1 or D2.

caused the quantum of energy to enter the front door of the interferometer as a wave or a particle. Cause and effect are reversed in time. The random number generator and state of the EOM was the "cause" whereas the earlier decision of the photon as it entered the front door of the interferometer was the "effect."

Therefore, QM believes it has PROVED that the quantum world is weird. The journal Science which published this study, is one of the two top scientific journals in the world.

The proof of their allegation is that when they look for a wave, they find a wave, and when they look for a particle they find a particle. The random choice which way to test the quantum was made AFTER the quantum had entered the front door of the interferometer, but BEFORE it reached the output area.

\subsubsection{Critique of the experiment of Vincent Jacques, et. al.}

We claim that R-propagators start at the detectors D1 and D2 and go backwards through the interferometer. Every quantum that enters the interferometer's front door (lower left in Figure 15) becomes a photon particle, and every photon travels on only one beam of the interferometer. Every time a detector clicks it is because a photon hits it. If a wave hits a detector without a photon, there will be no click.

Think about it! If every time you look for a wave you find a wave, and every time you look for a particle you find a particle, that proves that BOTH waves and particles are ALWAYS present. The equipment was so designed that if you found a particle you could not simultaneously test whether a wave was present, nor vice versa. To say that the quanta were either waves or particles, was an ASSUMPTION on the part of Jacques et al. That assumption was never tested.

\subsubsection{Camera metaphor}

This experiment can be explained if we use a metaphor. Imagine that you have a camera with which you take pictures of crowds of people. It has two lenses. One lens only sees women, not men. The other lens only sees men, not women. Without looking through the camera you cannot see anyone. 


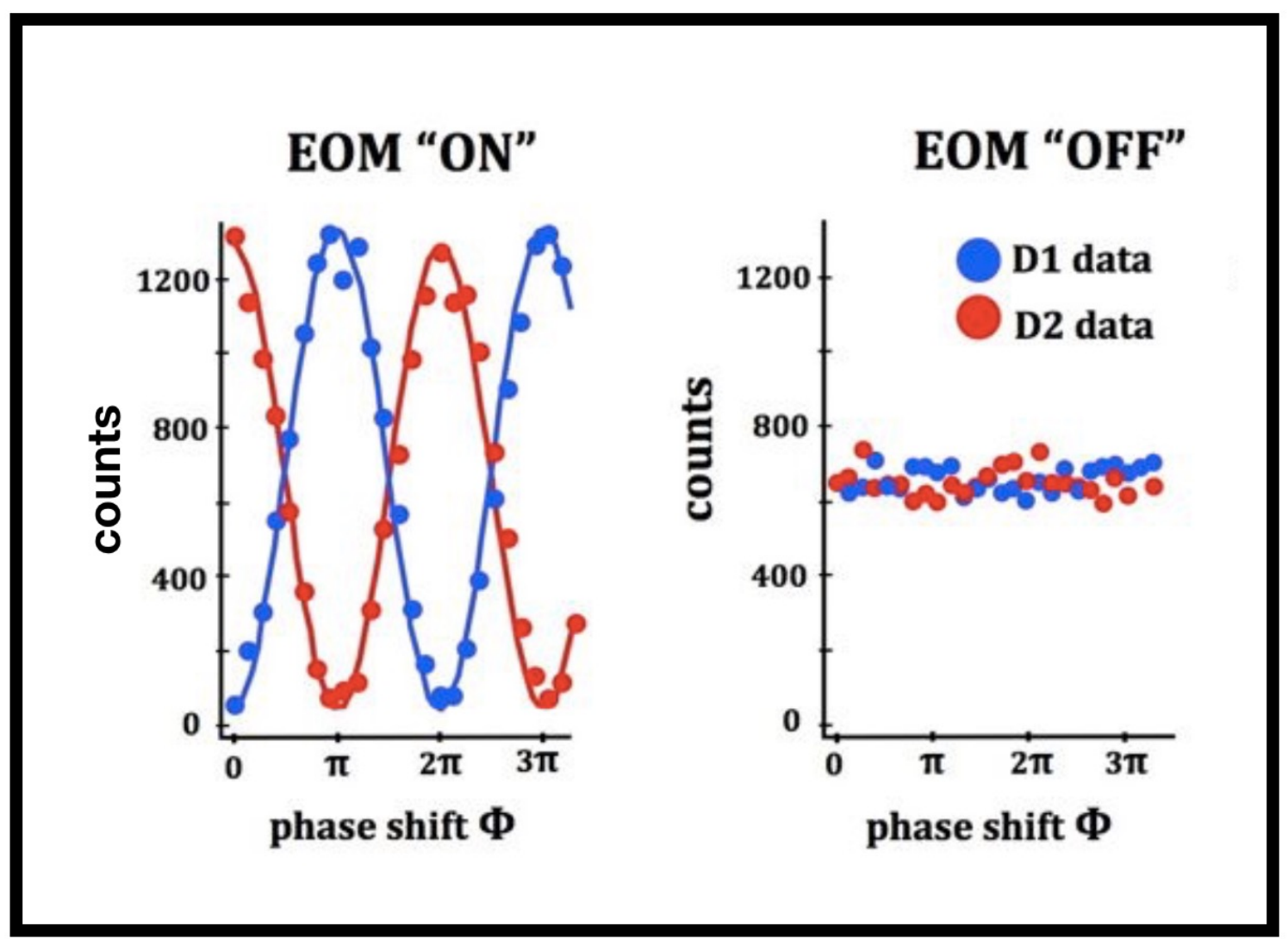

Figure 18: Output data upon which the entire Jacques experiment rests. On the left the equation is $Y \approx \pm 650 \times$ $\left(\cos ^{2} \phi\right)+700$. On the right it is $Y \approx 700 \pm 70$. When the EOM is "ON" they are testing for waves, and the sinusoidal waves on the left show that they see waves. When the EOM is "OFF" they are testing for particles, and the pattern on the right shows that they see particles.

So you take pictures with the camera and the pictures only show women. Then you changes lenses and the pictures only show men. You find this astounding: that the women are changing into men and the men are changing into women. It is obvious that your choice of lens is causing the people to change from female to male, or vice versa.

So you decide to change lenses more quickly to see if you can catch this transition before it occurs. You manage to change lenses at random in $40 \mathrm{~ns}$, whereas it takes $120 \mathrm{~ns}$ for photons from the crowd of people to reach your camera. Once again you discover that one lens shows women only, whereas the other lens shows men only. The conclusion that the journal Science drew is that your random choice of lens causes the people to change gender, and that this cause and effect goes backwards in time: first the change of gender, then the random choice of lens. This allegedly proved backwards in time cause and effect, which was what Wheeler predicted.

The cause was your change of lens. The effect was a change of gender, earlier in time.

This author thought this was illogical. So Ihe author wrote a scholarly letter to the editor and sent it to Science. In fifteen minutes the editor wrote an email back, "Not the kind of thing we publish."

\section{Conclusion}

This article has given you the equations that define an R-propagator: Eqs. 19-21. 


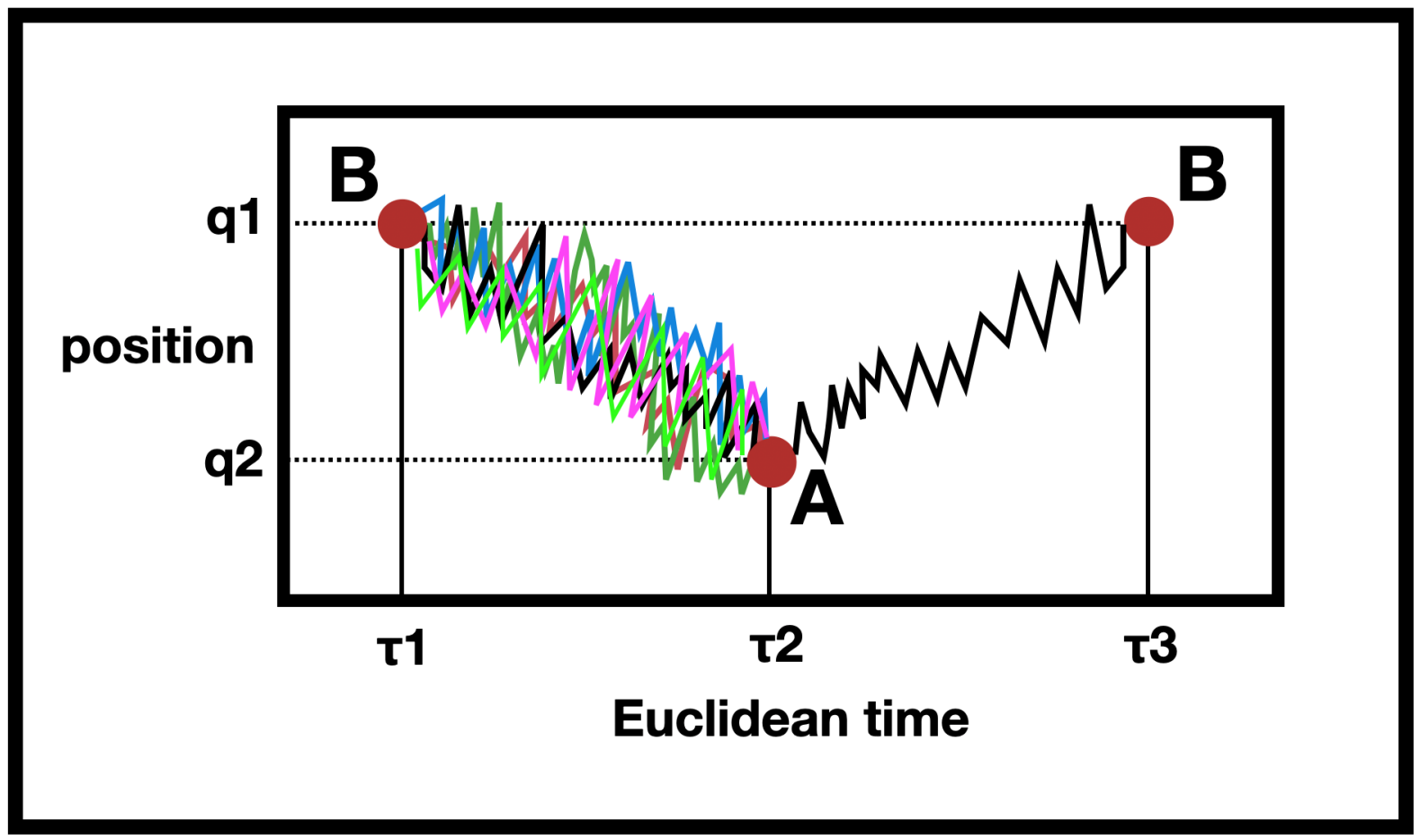

Figure 19: Our final model is shown on the left side of this graph, governed by Eqs. 19-21. However, the right side of the graph is different. The particle as it leaves the gun at point A is free to select any trajectory at random. It travels to the upper right to detector B. That unpredictable, whimsical freedom of the particle is not part of Feynman's model.

The action for all trajectories from $\tau_{1}$ to $\tau_{2}$ in Euclidean time (Fig. 19 left) is:

$$
\mathbf{S}_{E}[q]=-\int_{\tau_{1}}^{\tau_{2}} d \sigma\left[\frac{m}{2}\left(\frac{d q}{d \sigma}\right)^{2}+V\left(q_{\sigma}\right)\right] .
$$

$\mathbf{R}$-Propagator $=\mathbf{R}$-Kernel $=\mathbf{K}_{E}\left(q_{2}, \tau_{2}, q_{1}, \tau_{1}\right)=\left\langle q_{2}\right|\left(U_{E}\left(\tau_{2}-\tau_{1}\right)\left|q_{1}\right\rangle=\right.$

$$
\text { Euclidean path integral }=\int_{\tau_{1}}^{\tau_{2}} \mathcal{D}[q(\sigma)] \exp \left(-\frac{i}{\hbar} S_{E}(q)\right)
$$

The phase of the R-propagator is the central focus of attention. The exponent

$$
\exp \left(-\frac{i}{\hbar} \mathbf{S}_{E}(q)\right)
$$

is determined by the action $\mathbf{=} \mathbf{S}$.

\subsection{The particle is NOT "free of constraints" as it travels from $\mathbf{A}=\left(\tau_{2}, q_{2}\right)$ to $\mathbf{B}=\left(\tau_{3}, q_{1}\right)$ !}

Nevertheless, the particle is not free to choose any pathway to travel from A to B. For example, two successive particles would be unlikely to travel on precisely the same path. It would be inconceivable for three particles in succession to traverse the exact same path. The word "random" is notoriously mysterious. It appears to mean that the particle is free of all constraints. But as we watch 100 particles choose "random" trajectories, we discover that the particle is covertly constrained to follow a pattern. What pattern? A random pattern.

This mystery is central to the medical field. As they search for a medicine or vaccine to treat COVID-19, everything hinges on this word "random." The only medicine they will trust will be be one which has been tried in double blind controlled clinical trials, and found to be more effective in the treated group than in the control group at a probability of less than one in one hundred. That means that researchers are able to define "random" with sufficient accuracy that they are able to say, "If the experiment were run one hundred times, only in one of those experiments would you expect this peculiar pattern in the data." 
This mystery that governs the particle as it travels from $\mathrm{A}=\left(\tau_{2}, q_{2}\right)$ to $\mathrm{B}=\left(\tau_{3}, q_{1}\right)$ means that our model is precisely the same as Feynman's model, when it comes to the behavior of the particle. Fig. 19 is radically different than anything Feynman ever drew, and yet, when we watch Nature, we can't see any difference.

\subsection{Paths are bi-directional}

R-propagators can be photographed. You have seen two pictures of them in this article (Fig. 12 far right and Fig. 20). That is what you see on the screen of a double slit experiment. It is called an "interference fringe pattern" because it is the fingerprint of the interference that occurs proximate to the particle gun.

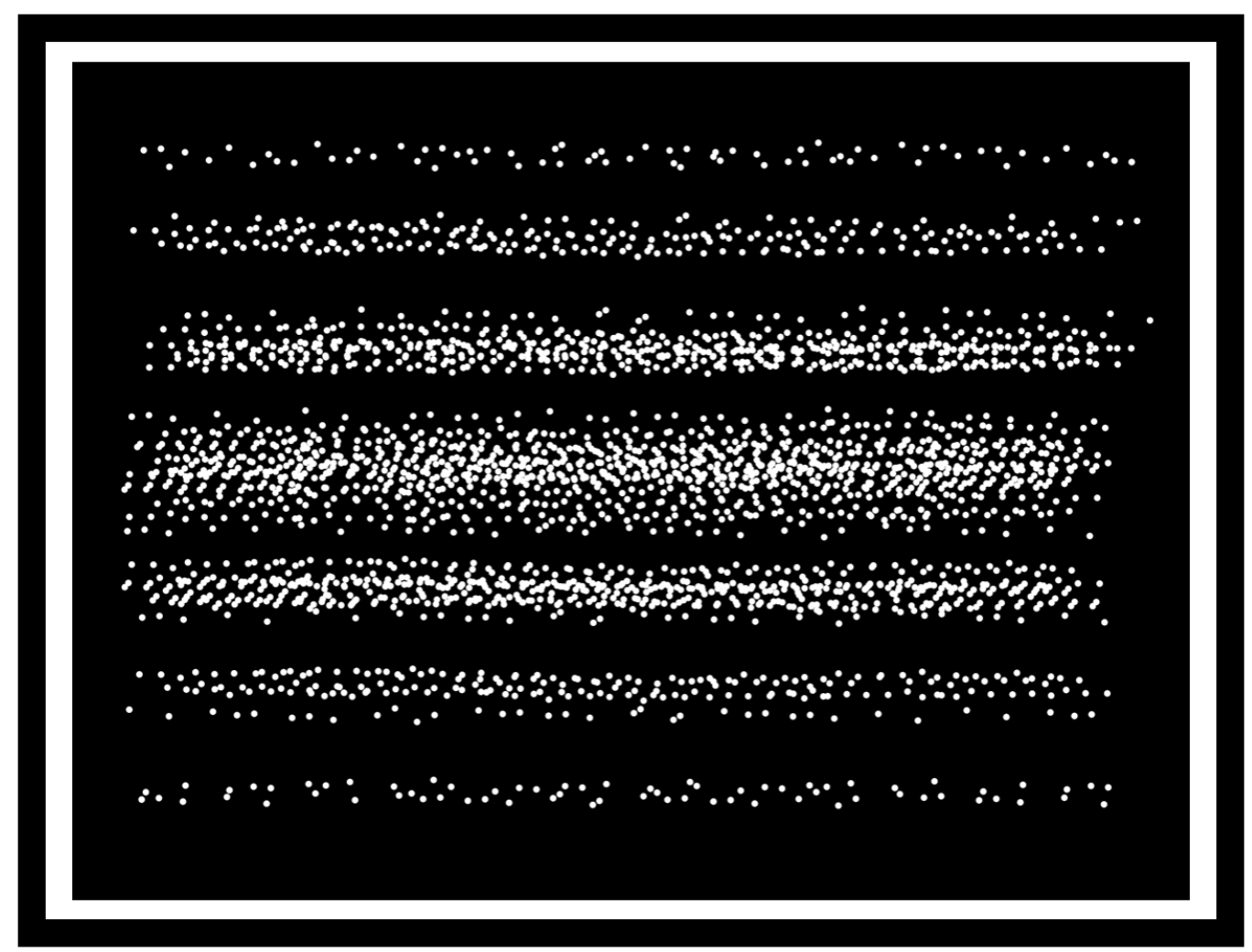

Figure 20: A picture of R-propagators impinging on the particle gun.

The mathematics of R-propagators and propagators is the same. QED could be run with either engine. The question is, "Which engine does Nature use?" There is abundant evidence from experiments cited above that Nature uses R-propagators, not propagators.

Consider a map of paths in the words. Some paths are broad and well traveled. Others are narrow and crooked. If we wanted to go across the woods in the least amount of time, we would be interested in finding the path with the least action. Something like this might have been in Feynman's mind when he chose the metaphor "paths" as the name of his mathematical model.

One thing Feynman forgot to consider: The same path can be traveled in either direction. The path itself has no directionality. All the energy for your hike comes from you, none from the path. If you meander from A to B, you can meander from B to A. On a practical level, traveling in the conventional direction lands you in a thicket, whereas traveling from B to A involves no thicket.

\section{Biography of the Author}

The author was born in 1943 in New Jersey, USA. His father was a factory worker who never graduated from elementary school. Boyd helped his father dig out the basement of a house by hand, with a pick, shovel and wheelbarrow. That is where he learned the meaning of perseverance. He was the first member of his family to go to college. He excelled in mathematics and was encouraged by his older cousin, Lewis E. Little to study applied mathematics at Brown University, which he did, graduating in 1965. As a follower of Dr. Martin Luther King Jr. he risked his life in the Civil Rights 


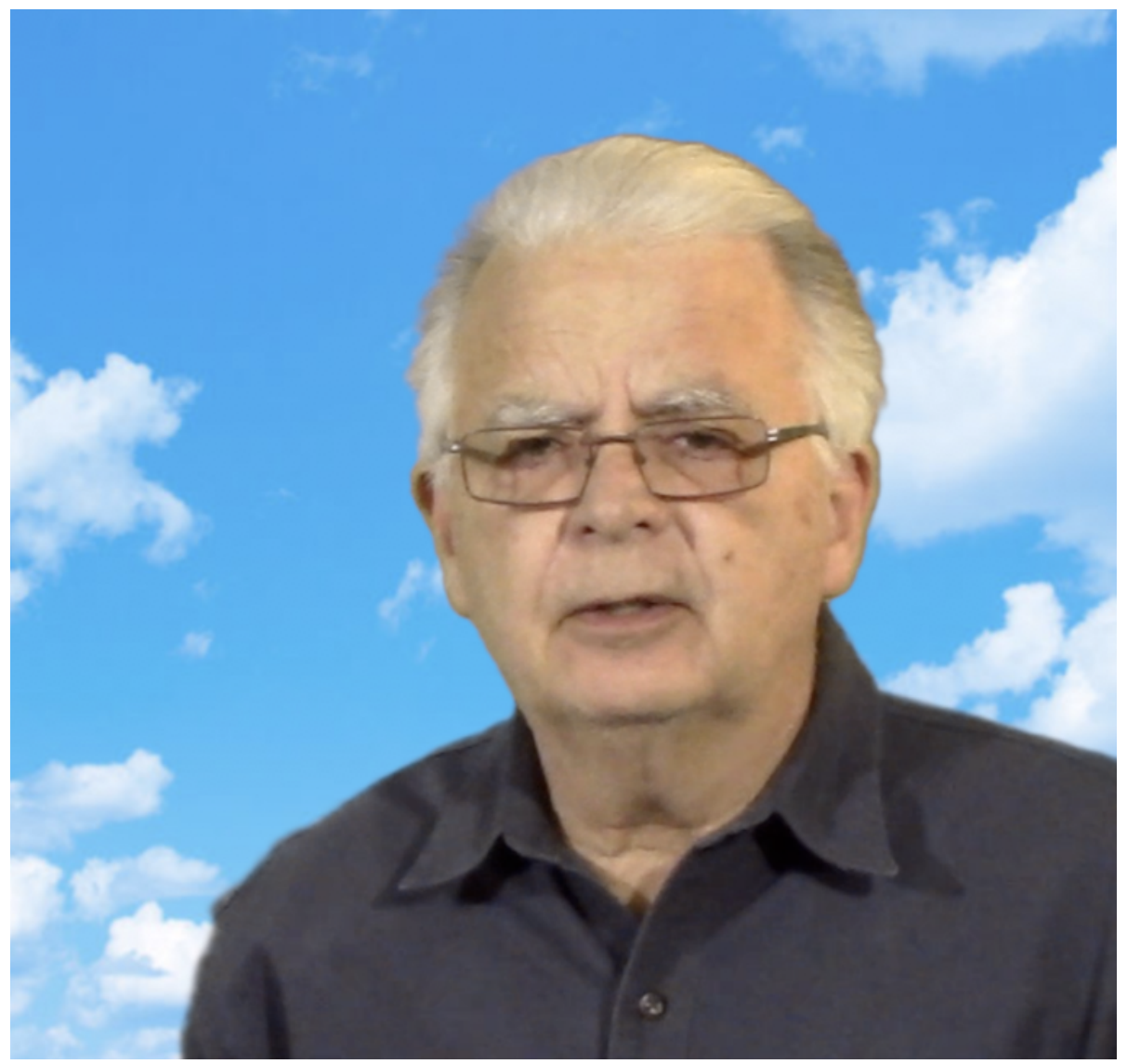

Figure 21: Jeffrey H. Boyd in 2020.

Movement. He subsequently earned graduate school degrees from Harvard University, Yale University, and Case Western Reserve Univesity. During medical school he treated patients who had survived the 1918 Spanish flu pandemic in which 25 million people died, then they were hospitalized for Parkinson's disease six decades after the Spanish flu virus had silently damaged the substantia nigra cells their brains. He became a physician in 1976. For a decade he was on the faculty of the National Institutes of Health in Bethesda, Maryland. Throughout his medical career his passion was for treating indigent people with chronic illnesses. For another decade he was Chairman of Ethics and Chairman of Behavioral Health at Waterbury Hospital in Connecticut. In the year 2000 at a birthday party for his aunt, held at Lewis Little's house, the author heard for the first time about his cousin's theory, the Theory of Elementary Waves (TEW). Starting in 2010 Boyd joined and began to speak at American Physical Society conventions, promoting TEW. Then he wrote scholarly articles published in peer reviewed physics and mathematics journals. In 2013 he and Little had a dispute and have not communicated since. Boyd has a website, ElementaryWave.com, which provides access to his many YouTube videos and scholarly articles on this subject.

\section{Acknowlegement}

The author thanks Lewis E. Little for teaching him TEW and asking him to build a mathematics.

\section{Conflict of interest}

None.

\section{References}

[1] Bloch, F. (1957) Generalized theory of relaxation. Physical Review, 1051206 DOI:https://doi.org/10.1103/PhysRev.105.1206 
[2] Boyd, J. H. (2020a) A tiny, counterintuitive change to the mathematics of the Schrődinger wave packet and Quantum ElectroDynamics could vastly simplify how we view Nature. Journal of Advances in Physics 17, 169-203. https://doi.org/10.24297/jap.v17i.8696

[3] Boyd, J. H. (2020b) New Schrődinger wave mathematics changes experiments from saying there is, to denying there is quantum weirdness. Journal of Advances in Mathematics 18, 82-117. https://doi.org/10.24297/jam.v18i.8656

[4] Boyd, J. H. (2020c) New Schrôdinger wave math changes experiments so they deny there is quantum weirdness. https://www.youtube.com/watch?v=_k9aDgDYUco\&feature=youtu.be (access date 2020-02-24).

[5] Boyd, J. H. (2020d) A mathematical explanation for the double slit experiment of quantum mechanics. https://www.youtube.com/watch?v=O9dpDcF6Uhs (access date 2020-02-24).

[6] Boyd, J. H. (2019a) Decrypting the central mystery of quantum mathematics: Part 1. New axioms explain the double slit experiment. Journal of Advances in Mathematics 17, 255-282. https://doi.org/10.24297/jam.v17i0.8475

[7] Boyd, J. H. (2019b) Decrypting the Central Mystery of Quantum Mathematics: Part 2. A mountain of empirical data supports TEW. Journal of Advances in Mathematics 17, 283-314. https://doi.org/10.24297/jam.v17i0.8489

[8] Boyd, J. H. (2019c) Decrypting the central mystery of quantum mathematics: Part 3. A non-Einstein, non-QM view of Bell test experiments. Journal of Advances in Mathematics 17, 315-331. https://doi.org/10.24297/jam.v17i0.8490

[9] Boyd, J. H. (2019d) Decrypting the central mystery of quantum mathematics: Part 4. In what medium do Elementary Waves travel? Journal of Advances in Mathematics 17, 332-351. https://doi.org/10.24297/jam.v17i0.8491

[10] Boyd, J. H. (2018a) The von Neumann and double slit paradoxes lead to a new Schrôdinger wave mathematics. Journal of Advances in Physics 14, 5812-5834. https://doi.org/10.24297/jap.v14i3.7820

[11] Boyd, J. H. (2018b) The quantum world is astonishingly similar to our world: The timing of wave function collapse according to the Theory of Elementary Waves. Journal of Advances in Physics 14, 5598-5610. DOI: 10.24297/jap.v14i2.7555

[12] Boyd, J. H. (2017) A symmetry hidden at the center of quantum mathematics causes a disconnect between quantum math and quantum mechanics. Journal of Advances in Mathematics, 13, 7379-86. DOI: 10.24297/jam.v13i4.6413.

[13] Boyd, J. H. (2017) Paul Dirac's view of the Theory of Elementary Waves. Journal of Advances in Physics 13, 4731-4734. DOI: https://doi.org/10.24297/jap.v13i3.5921

[14] Boyd, J. H.(2017) The Boyd Conjecture. Journal of Advances in Physics 13, $4830-37$. https://doi.org/10.24297/jap.v13i4.6038 (access date 2020-02-27)

[15] Boyd, J. H. (2015a) A paradigm shift in mathematical physics, Part 4: Quantum computers and the local realism of all 4 Bell states. Journal of Advances in Mathematics, 11, 5476-5493. https://doi.org/10.24297/jam.v11i7.1224.

[16] Boyd, J. H. (2015b) A paradigm shift in mathematical physics, Part 3: A mirror image of Feynman's quantum electrodynamics (QED. Journal of Advances in Mathematics, 11, 3977-3991. DOI: https://doi.org/10.24297/jam.v11i2.1283.

[17] Boyd, J. H. (2015c) A paradigm shift in mathematical physics, Part 2: A new local realism explains Bell test \& other experiments. Journal of Advances in Mathematics, 10, 3828-3839. DOI: https://doi.org/10.24297/jam.v10i9.1884.

[18] Boyd, J. H. (2015d) A paradigm shift in mathematical physics, Part 1: The Theory of Elementary Waves (TEW). Journal of Advances in Mathematics 10, 3828-3839. http://cirworld.com/journals/index.php/jam/article/view/4719. (access date 2020-02-27)

[19] Boyd, J. H. (2015e) The Theory of Elementary Waves eliminates Wave Particle Duality. Journal of Advances in Physics 7, 1916-1922. https://www.rajpub.com/index.php/jap/article/view/2279. (access date 2020-02-27)

[20] Boyd, J. H. (2015f) A new variety of local realism explains a Bell test experiment: the Theory of Elementary Waves (TEW) with no hidden variables'. Journal of Advances in Physics 8 , 2051-58. https://www.semanticscholar.org/paper/A-new-variety-of-local-realism-explains-a-Bell-testBoyd/445009d95dd80180537216f953dbf4d4ddc8af7d. (access date 2020-02-27)

[21] Boyd, J. H. (2015g) A proposed physical analog of a quantum amplitude: Corkscrew model from the Theory of Elementary Waves (TEW). Journal of Advances in Physics 10, 2774-2783. https://rajpub.com/index.php/jap/article/view/1324 (access date 2020-02-27)

[22] Boyd, J. H. (2013a) Re-thinking a delayed choice quantum eraser experiment: a simple baseball model. Physics Essays, 26, 100-109. DOI: 10.4006/0836-1398-26.1.100.

[23] Boyd, J. H. (2013b) Re-thinking Alain Aspect's 1982 Bell test experiment with delayed choice. Physics Essays, 26, 582-591. https://doi.org/10.4006/0836-1398-26.4.582

[24] Boyd, J. H. (2012) Rethinking a Wheeler delayed choice gedanken experiment. Physics Essays 25, $390-396$. https://doi.org/10.4006/0836-1398-25.3.390

[25] Carroll, L. (1871) Through the Looking Glass, Altenmúnster, Germany: Jazzybee Verlag Júrgen Beck. ISBN 9783849621742 
[26] Davisson, C. J. and L. Germer (1927) Reflection of electrons by a crystal of nickel Nature, 119 558-560. https://doi.org/10.1038/119558a0

[27] Davisson, C.J.,(1928a) The diffraction of electrons by a crystal of nickel Bell System Technical Journal 7 90-105. https://doi.org/10.1002/j.1538-7305.1928.tb00342.x

[28] Davisson, C. J. (1928b) Are Electrons Waves? Franklin Institute Journal 205, 597. https://doi.org/10.1016/S00160032(28)90979-5

[29] Dotson, A. (2019) What a path integral problem looks like in quantum mechanics. https://www.youtube.com/watch?v=dCkHDMsb33U (accessed 2020-04-24).

[30] Feynman, R. P. (2010) Feynman Lectures on Physics, vol. 3 (Basic Books) ISBN-13: 978-0465025015, see pages I-1 to 1-11 discussion of the double slit experiment as the central mystery of QM.

[31] Feynman, R. P. (1985) QED: The Strange Theory of Light and Matter (Princeton University Press). ISBN 978-0-691-12575-6

[32] Feynman, R. P. (c1964-2013) The principle of least action. https://www.feynmanlectures.caltech.edu/II_19.html (accessed 2020-04-24).

[33] Feynman, R. P. and A. R. Hibbs (c1965) Quantum Mechanics and Path Integrals (Mineola, NY: Dover Publications). ISBN-13 978-0-468-47722-0.

[34] Freire, O. (2003) Interview with Dr. Franco Selleri. American Institute of Physics: Oral History Interviews. https://www.aip.org/history-programs/niels-bohr-library/oral-histories/28003-1. and https://www.aip.org/historyprograms/niels-bohr-library/oral-histories/28003-2. (accessed July 15, 2019)

[35] Jacques, V., E. Wu, F. Grosshans, et. al. (2007) Experimental realization of Wheeler's delayed-choice gedanken experiment Science, 315, 966-968. DOI: 10.1126/science.1136303

[36] Jacques, V., E. Wu, T. Toury, et. al. (2005) Single-photon wavefront-splitting Interference European Physical Journal D, 35, 966-968 561-565. DOI: 110.1140/epjd/e2005- 00201-y

[37] Kaiser, H., R. Clothier, S. A. Werner, et. al. (1992) Coherence and spectral filtering in neutron interferometry, Physical Review 45, 31-42. https://doi.org/10.1103/PhysRevA.45.31

[38] Little, L. E. (1996) Theory of Elementary Waves. Physics Essays 9, 100-134. https://doi.org/10.4006/1.3029212

[39] Little, L. E. (2000) Theory of Elementary Waves. Lecture at the Jet Propulsion Labs. Go to YouTube and search for "Lewis E. Little JPL" (access date Feb 17, 2020).

[40] Little, L. E. (2009) Theory of Elementary Waves. New Classics Library, New York. ISBN: 978-161694-032-1

[41] Pfleegor, R. L. and L. Mandel. (1967) Interference of independent photon beams. Physical Review, 159,1084-1088. https://doi.org/10.1103/PhysRev.159.1084

[42] Pfleegor, R. L. and L. Mandel. (1968) Further experiments on interference of independent photon beams at low light levels. Journal of the Optical Society of America, 58, 946-950. https://doi.org/10.1364/JOSA.58.000946

[43] Raviteja, K.(2017) "Euclidean time and field theory, path integrals" https://www.youtube.com/watch?v=v_FmiMaiciA (access date 2020-04-19).

[44] Scully, M. O. and K. Drühl. (1982) Quantum eraser: A proposed photon correlation experiment and 'delayed choice' in quantum mechanics. Physical Review A, 25, 2208-2213. https://doi.org/10.1103/PhysRevA.25.2208

[45] Wheeler, J. A. and R. P. Feynman (1945) Interaction with the absorber as the mechanism of ratiation. Reviews of Modern Physics, 17. 157-181. https://doi.org/10.1103/RevModPhys.17.157 